Review

\title{
Current and Prospective Radiation Detection Systems, Screening Infrastructure and Interpretive Algorithms for the Non-Intrusive Screening of Shipping Container Cargo: A Review
}

\author{
Euan L. Connolly *(i) and Peter G. Martin (1)
}

check for updates

Citation: Connolly, E.L.; Martin, P.G. Current and Prospective Radiation Detection Systems, Screening Infrastructure and Interpretive Algorithms for the Non-Intrusive Screening of Shipping Container Cargo: A Review. J. Nucl. Eng. 2021, 2, 246-280. https://doi.org/10.3390/ jne2030023

Academic Editor: Dan Gabriel Cacuci

Received: 25 May 2021

Accepted: 29 July 2021

Published: 7 August 2021

Publisher's Note: MDPI stays neutral with regard to jurisdictional claims in published maps and institutional affiliations.

Copyright: (c) 2021 by the authors. Licensee MDPI, Basel, Switzerland. This article is an open access article distributed under the terms and conditions of the Creative Commons Attribution (CC BY) license (https:/ / creativecommons.org/licenses/by/ $4.0 /)$.
Interface Analysis Centre, HH Wills Physics Laboratory, School of Physics, University of Bristol, Tyndall Avenue, Bristol BS8 1TL, UK; peter.martin@bristol.ac.uk

* Correspondence: e.connolly@bristol.ac.uk

\begin{abstract}
The non-intrusive screening of shipping containers at national borders serves as a prominent and vital component in deterring and detecting the illicit transportation of radioactive and/or nuclear materials which could be used for malicious and highly damaging purposes. Screening systems for this purpose must be designed to efficiently detect and identify material that could be used to fabricate radiological dispersal or improvised nuclear explosive devices, while having minimal impact on the flow of cargo and also being affordable for widespread implementation. As part of current screening systems, shipping containers, offloaded from increasingly large cargo ships, are driven through radiation portal monitors comprising plastic scintillators for gamma detection and separate, typically ${ }^{3} \mathrm{He}$-based, neutron detectors. Such polyvinyl-toluene plastic-based scintillators enable screening systems to meet detection sensitivity standards owing to their economical manufacturing in large sizes, producing high-geometric-efficiency detectors. However, their poor energy resolution fundamentally limits the screening system to making binary "source" or "no source" decisions. To surpass the current capabilities, future generations of shipping container screening systems should be capable of rapid radionuclide identification, activity estimation and source localisation, without inhibiting container transportation. This review considers the physical properties of screening systems (including detector materials, sizes and positions) as well as the data collection and processing algorithms they employ to identify illicit radioactive or nuclear materials. The future aim is to surpass the current capabilities by developing advanced screening systems capable of characterising radioactive or nuclear materials that may be concealed within shipping containers.
\end{abstract}

Keywords: nuclear; proliferation; RDD; INED; radiation; infrastructure; monitoring; security; ports; material smuggling

\section{Introduction}

In the United Kingdom (UK), the screening of shipping containers for concealed nuclear and/or radioactive materials has been implemented across all of the country's container cargo ports in response to the September 11th 2001 terrorist attacks in the United States of America (USA). It was following this pivotal series of events that the threat posed by terrorist and extremist groups obtaining and using even small quantities of nuclear or radioactive material was realised [1,2]. Deterring and detecting the illicit transport of nuclear or radioactive materials is essential, as special nuclear materials can be used to create improvised nuclear explosive devices (INEDs) and radioactive materials can be used to construct radiological dispersal devices (RDDs). Such a need for nations to implement monitoring capabilities, typically at their borders, has long been identified as a crucial part of security infrastructure by the International Atomic Energy Agency (IAEA) [3]. Special nuclear materials (SNMs) are any substance that could be used to 
produce a nuclear weapon, such as ${ }^{239} \mathrm{Pu},{ }^{233} \mathrm{U}$, highly enriched uranium (HEU), depleted uranium (DU), thorium, as well as other fissile or fissionable isotopes [3-5]. In contrast to complex and highly engineered atomic or thermonuclear bombs, RDDs or "dirty bombs" are very simplistic, comprising an explosive device that disperses radioactive material upon detonation. Whereas the detonation of a nuclear weapon seeks to cause significant physical destruction and immediate loss of life, an RDD serves to contaminate an area with radioactive materials, instill civil unrest and necessitate a considerable post-event response $[2,6]$. While it is possible to use any radioactive material in the construction of a disorder-inducing RDD, certain radioisotopes are identified by the IAEA to pose the highest security concern based on the energy and type of radiation they emit, their half-life $\left(t_{1 / 2}\right)$ being on the scale of human lifetimes, and their availability, with isotopes meeting these criteria including ${ }^{60} \mathrm{Co},{ }^{137} \mathrm{Cs},{ }^{192} \mathrm{Ir}$ and ${ }^{241} \mathrm{Am}[2,7,8]$.

Introduced in 1995 as part of the IAEA Nuclear Security Plan [3], the Incident and Trafficking Database (ITDB) has collated reports of events relating to the trafficking or malicious use of illicit radioactive materials since 1993 [9]. While only six confirmed events of trafficking or "use with malicious intent" were reported in 2019, as shown in Figure 1, the potentially catastrophic consequences of the detonation of nuclear weapons, INEDs or RDDs warrants the continued efforts to detect and deter illicit activities using nuclear or radioactive materials. The ITDB confirms that nuclear and radioactive materials out of regulatory control are attainable by groups or individuals, and the continued occurrences of these events indicates that there is perceived, if not real, demand for these materials by those seeking to cause damage and harm [9]. Resulting from an increase in the global amount of nuclear and radioactive materials owing to an international trend towards nuclear power generation as a low-carbon energy source, there are consequently expanding opportunities for the loss or theft of nuclear and radioactive materials [10]. So now, more than ever, there is the critical need to keep such material under strict regulatory control and prevent its illicit trafficking within an increasingly unstable and unpredictable world through robust border monitoring capabilities.

Sea freight comprises the majority of global cargo transportation, and as a historic island trading nation, approximately $95 \%$ of the UK's imports and exports (by weight) are transported in this way. Therefore, screening cargo at seaports forms an essential mechanism for detecting the illicit cross-border movement of nuclear and/or radioactive materials into the UK [11]. Not including bulk materials such as oil, gas and mineral/metallic ores (which make up the majority of UK ports imports by mass), containerised freight represented $43 \%$ (by mass) of UK port imports in 2019, with the remaining $57 \%$ comprised of "roll-on roll-off" (Ro-Ro) freight, such as live animals, motor vehicles and road goods vehicles [11]. As seaports serve as natural bottlenecks for freight, both departing and entering a country, they represent an obvious location for the installation of screening systems. In the UK, as in many other countries around the world, radiation portal monitors (RPMs) are utilised at seaports to screen both containerised and Ro-Ro freight. Looking towards the future, in its Strategy for Countering Terrorism the UK government has identified the need for refreshing its radiological and nuclear deterrence alongside its detection capabilities at its international borders by developing "enhanced innovative detection technologies" [12].

In order to identify nuclear and radioactive materials, screening systems utilise radiation detectors that are sensitive to the penetrating neutrons and gamma-rays ( $\gamma$-rays) emitted during a materials radioactive decay. Data processing algorithms then interpret the number of radiation interaction events measured by detectors to assert whether nuclear or radioactive material is present. Currently, the most common detection systems in use for shipping container screening are comprised of polyvinyl-toluene (PVT) scintillator panels in RPMs positioned around single traffic lanes. These large-volume systems serve to flag containers for further inspection based on the gross number of radiation detection events when compared to the number of background events in specific energy windows, which are chosen to allow for the discrimination between different types of nuclear or radioactive materials that emit radiation in characteristic energy regions [13]. This system 
achieves acceptable detection sensitivity and false alarm rates, which together are the basic requirements of a shipping container screening system - with the lane-straddling arrays possessing the further benefit of having minimal impact on transportation throughput, as containers can be driven directly through the RPM setup. An additional benefit comes from being of low cost-a key factor in permitting nationwide implementation. The major drawback of this system, however, is that the low energy resolution of PVT detectors necessitates a secondary inspection step to validate all alarms in order to characterise and locate any nuclear/radioactive materials responsible for the trigger event. Ideally, the next generation of such shipping container screening systems should be comparable in throughput and cost to existing systems, but possess the enhanced capabilities necessary to identify the isotopic composition of nuclear or radioactive materials and localise their positions within a container in a single, unified, inspection process. This will ultimately expedite the screening process, reduce false-positive occurrences and eliminate the need for an inefficient secondary inspection phase.

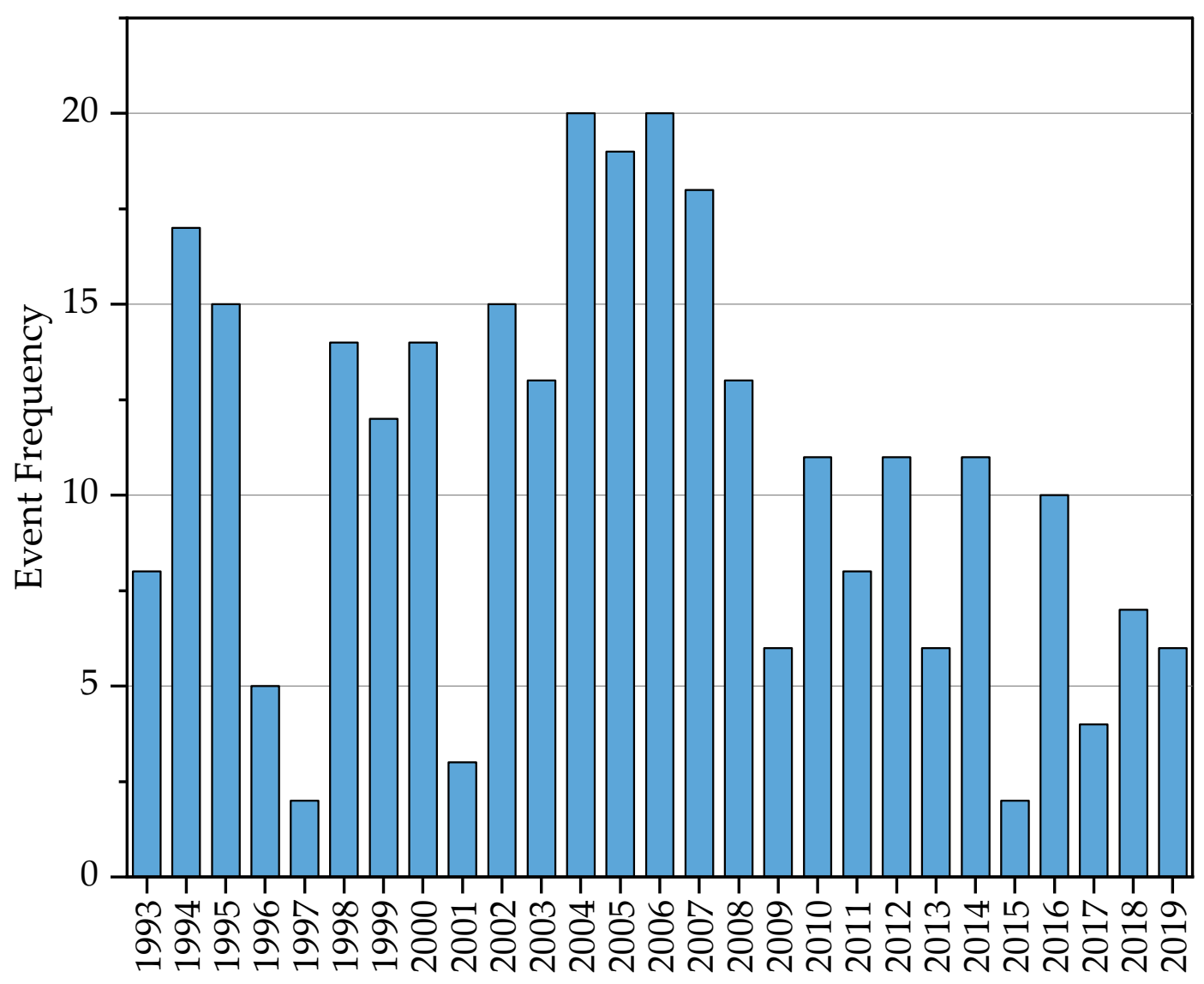

Figure 1. Plot detailing the number of events reported to the ITDB which relate to the trafficking or malicious use of illicit nuclear or radioactive materials between 1993 and 2019. Reprinted, with permission, from the International Atomic Energy Agency-IAEA, www.iaea.org (accessed on 29 July 2021) [9].

As alluded to above, one of the challenges faced by existing shipping container screening systems at seaports is the prevention and mitigation of false alarms that occur primarily due to the transportation of naturally occurring radioactive materials (NORMs) and, to a much lesser extent, due to variations in natural background [14-16]. When such false-positive trigger events occur, the identified containers subsequently require further inspection-resulting in delays to the onwards transportation of freight. These variations in background radiation arise from the material properties of the detector surroundings (e.g., concrete types), as well as external factors such as weather and prevailing solar activity $[17,18]$. NORM sources are common imports such as ceramics, fertilizer and cat 
litter which contain radioactive isotopes appearing in nature (e.g. ${ }^{40} \mathrm{~K}$ and the uranium and thorium decay series) that trigger false-positive alarms as the screening system measures a spike in radiation detection events $[15,16]$. Radioactive isotopes used for medical or commercial purposes that have not been appropriately declared are another source of false alarms; one such medical isotope is ${ }^{99 \mathrm{~m}} \mathrm{Tc}$, which is the radioisotope most commonly used in medical procedures, constituting up to $87 \%$ of dosages in US hospitals in 2001 [19]. Dependent upon the type of radiation detector used, a range of data processing algorithms are available, or under development, for differentiating between legitimate and false alarms, as the rate of false alarms, along with its detection efficiency, are key metrics for assessing the performance of a screening system.

The discussion in this review is focused on the passive detection of radiation emitted by concealed radioactive sources. However, other techniques exist involving the active screening of freight with particle beams (e.g., X-rays, protons) or alternatively, cosmic muons [20]. Cosmic muon tomography is particularly useful for detecting SNMs as muons are scattered at larger angles by high atomic number $(Z)$ and density materials; by measuring the scattering angle, the presence of SNMs and other high Z materials can be identified [21]. Such a system has obvious benefits, with no equipment required to generate a particle beam and the capability to identify SNMs in containers in minutes [22]. However, a secondary system would be still required to identify low $\mathrm{Z}$ radioactive materials. Proton and $\mathrm{X}$-ray active interrogation systems operate on a similar principle to cosmic muon tomography but with the additional requirement of beam-generating infrastructure [23]. Xray radiography can additionally be used to screen cargo for contraband beyond radioactive materials, such as explosives, and can even be used to detect SNMs by induced fission, making it a promising candidate for widespread implementation [20]. In any case, these active or muon tomography screening systems are likely to be employed in conjunction with a passive detection system for identifying low $\mathrm{Z}$ radioactive materials and radioactive emission signatures, which are required for isotopic identification.

Within this review, existing and prospective screening systems for the non-intrusive inspection of shipping containers for concealed nuclear or radioactive materials will be discussed and their performance evaluated. Initially, the relevant theory associated with detecting radioactive emitters is introduced (Section 2) and applied to the detection of sources concealed within shipping containers (Section 3), with screening systems currently deployed and the mechanisms of assessing their performance discussed. Subsequently, in Section 4, the methods aimed at improving the performance of currently utilised RPMs are presented and evaluated. Finally, in Section 5, a review of emerging research applicable to the development of novel screening systems is provided, with the aim of identifying techniques likely to constitute components for incorporation within future non-intrusive shipping container inspection systems.

\section{Radiation Detection Principles and Methods}

\subsection{Properties of Radiation}

Unstable radioactive nuclei decay by emitting one or a number of ions such as alpha $(\alpha)$ particles or protons, beta $(\beta)$ particles (electrons or positrons), gamma $(\gamma)$ radiation (high-energy photons) or neutrons and it is the detection of these particles that allows radioactive material to be located and identified [24]. The intensity of emitted radiation, measured at distances away from a source depends on the type and energy of the radiation, the distance between the source and detector and the composition of the matter between them. Compared to $\alpha$ and $\beta$ radiation, with ranges of a few centimetres and metres in air, respectively, the range of $\gamma$ and neutron radiation in air (and other media) makes them suitable for the detection of distant or concealed sources.

Including interactions with matter that attenuate radiation, the measured intensity of radiation reaching a detector at a distance, $r$, away from a point source can be expressed as

$$
I(r) \propto \frac{I_{0} B \exp \left(-\int \mu(r) d r\right)}{r^{2}},
$$


where $I_{0}$ is the intensity at a $r=0, B$ is a dimensionless buildup factor to account for radiation scattering into the detector path and $\mu(r)$ is the material specific linear attenuation coefficient that is integrated over the path length (for a $1 \mathrm{MeV} \gamma$ in Air, Fe and $\mathrm{Pb}$ the linear attenuation coefficients are approximately $7.8 \times 10^{-5}, 0.18$ and $0.70 \mathrm{~cm}^{-1}$, respectively) [24,25]. As shown by the exponential term in Equation (1), any interaction between the $\gamma$ or neutron radiation and material along its path attenuates the signal that is resultantly incident onto the detector. In its simplest description, neutrons interact with nuclei in matter via mechanisms such as neutron capture and nuclear recoil to produce secondary particles varying from heavy charged ions to gammas $[24,26]$. Conversely, $\gamma$ radiation interacts with matter to produce electrons/positrons via three main mechanisms; the photoelectric effect, Compton scattering or pair production [24,27].

For both neutrons and $\gamma$ radiation, the degree of attenuation between the source and detector depends on the neutron or $\gamma$ energy and the material properties, specifically the interaction cross section. The total interaction cross section is the sum of the cross sections for different interaction mechanisms, and its dependence on neutron or $\gamma$ energy and material properties (e.g., Z, density) is shown in Figure 2 for $\gamma$ radiation in $\mathrm{Pb}$, an effective radiation shield that may be used to conceal illicit radioactive or nuclear material.

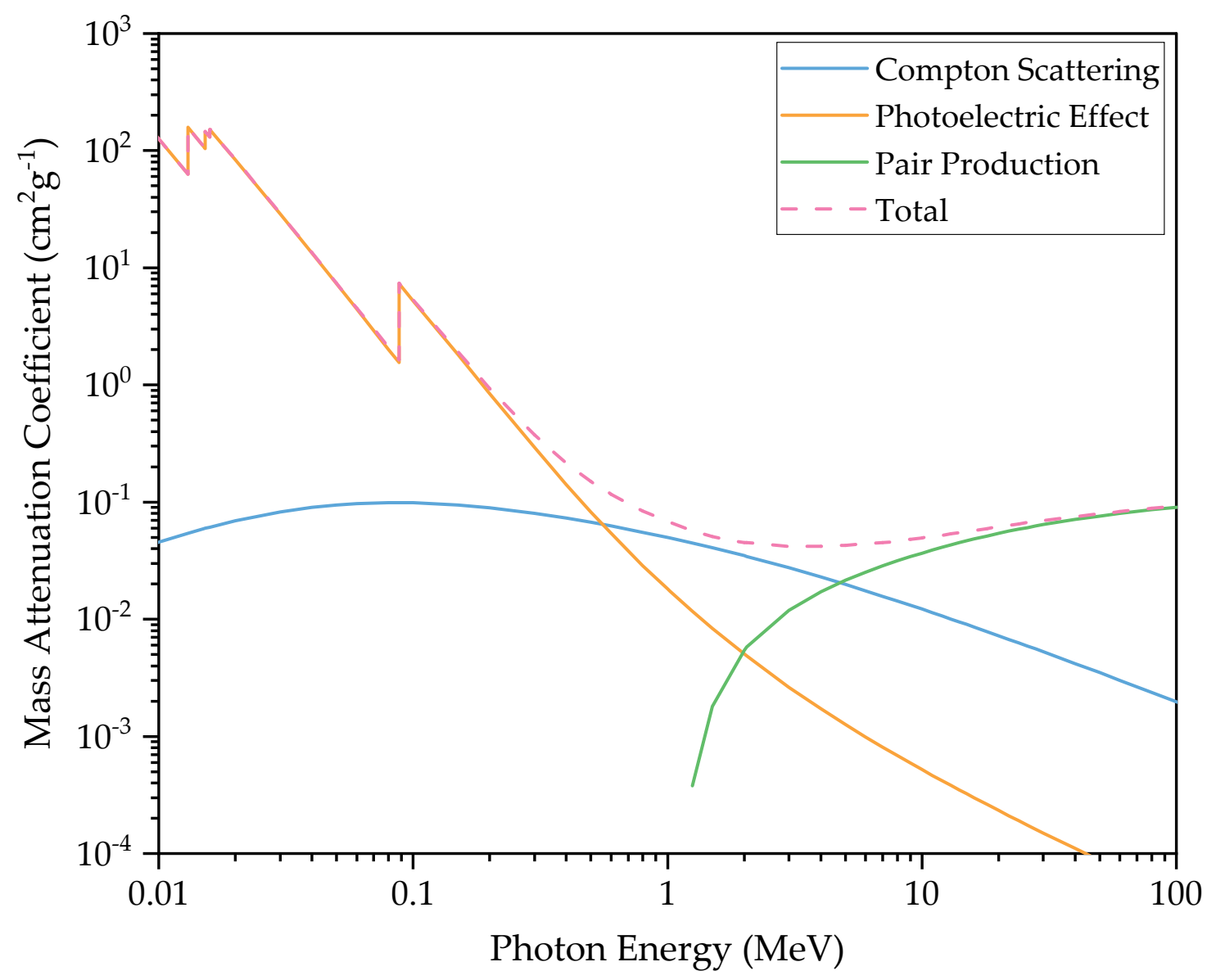

Figure 2. Mass attenuation coefficients in $\mathrm{Pb}$ for the $\gamma$ interaction mechanisms: Compton scattering (blue), photoelectric effect (orange), pair production (green) and total interaction cross section (pink) across the $10^{1}-10^{5} \mathrm{keV}$ energy range. Data from [25].

\subsection{Radiation Detectors}

By the same mechanisms as discussed in Section 2.1, neutrons and $\gamma$ radiation interact with materials in radiation detectors. The geometric efficiency of a detector is defined by the solid angle from the source subtended by the detector, representing the fraction of the total emission that is incident onto the detector. In contrast, the intrinsic efficiency of a detector 
for any type of radiation is the fraction of radiation incident on the detector that is detected, hence detectors which utilise materials with high linear attenuation coefficients have higher intrinsic efficiencies [27]. For thermal neutrons ( $<0.5 \mathrm{eV}$ [24]), the most important interaction process that enables detection is neutron absorption by nuclei with large neutron absorption cross sections at thermal energies (e.g., $\left.{ }^{3} \mathrm{He},{ }^{10} \mathrm{~B}\right)$. This leads to secondary radiation by radiative capture or induced fission. For fast neutrons $(>0.5 \mathrm{eV})$, the most important process is nuclear recoil, where neutrons transfer energy to nuclei by scattering interactions [28]. When an incident $\mathrm{n}$ or $\gamma$ interacts in a detector, the energy it transfers to particles in the material triggers a subsequent cascade of collisions, at the end of which remain electronhole (e-h) pairs, proportional in number to the energy deposited by the incident $\mathrm{n}$ or $\gamma[24,26]$. This mechanism describes the process of e-h production common to two types of solid detectors, semi-conductors and scintillators; how these different detectors measure the e-h pairs directly contributes to their response time and energy resolution. The energy resolution of a detector determines its ability to distinguish between incident radiation at different energies, and is defined by the full width at half maximum (FWHM) of a peak from a mono-energetic source as a percentage of the source energy [24].

Some of the earliest radiation detectors in widespread use were gas-based radiation detectors and while they still find use in specific applications such as ${ }^{3} \mathrm{He}$-based neutron detectors, solid detectors are favoured as they typically have better spectroscopic capabilities, are higher density and do not require high voltages and pressures to operate, making them more practical [24]. In recent years, there has been continued development and advancement of radiation detector materials - each with their own strengths and limitations. While semi-conductor detectors achieve high energy resolution for low energy $\gamma$ radiation, they are typically not suitable for use in shipping container screening due to the high cost and limited sizes of high-purity semi-conducting crystals available, operational requirements such as cooling and susceptibility to radiation damage [27,29]. For these reasons, the focus is on scintillator detectors, which can meet the cost, performance and operational requirements of a shipping container screening system. Properties of common scintillators are shown in Table 1.

Table 1. Properties of different organic (top) and inorganic (bottom) radiation detection materials used in scintillator detectors. Available sizes are based on typical commercial availability.

\begin{tabular}{|c|c|c|c|c|c|c|c|}
\hline Material & $\begin{array}{c}\mathrm{Z} \\
\text { (Effective Z) }\end{array}$ & $\begin{array}{l}\text { Density } \\
\left(\mathrm{g} \mathrm{cm}^{-3}\right)\end{array}$ & $\begin{array}{l}\text { Light Yield } \\
\text { (ph/keV) }\end{array}$ & $\begin{array}{c}\text { Energy Res. } \\
\text { (FWHM at } \\
662 \mathrm{keV)}\end{array}$ & $\begin{array}{l}\text { Available } \\
\text { Sizes }\end{array}$ & Notes & Refs. \\
\hline PVT & 1,6 & 1.02 & $\sim 10$ & - & $\sim \mathrm{m}^{2}$ & Plastic & {$[26,30]$} \\
\hline EJ-309 & 1,6 & 0.96 & 12.3 & - & & Liquid & {$[24,31]$} \\
\hline $\mathrm{NaI}(\mathrm{Tl})$ & $11,53,(56)$ & 3.67 & $38-55$ & $7.0 \%$ & $10^{3} \mathrm{~cm}^{3}$ & Hygroscopic & {$[24,26,32,33]$} \\
\hline CsI(Tl) & (54) & 4.51 & 54 & $<12 \%$ & $10^{3} \mathrm{~cm}^{3}$ & & {$[27,33,34]$} \\
\hline $\mathrm{LaBr}_{3}(\mathrm{Ce})$ & 35,57 & 5.29 & 63 & $2.7 \%$ & $10^{2} \mathrm{~cm}^{3}$ & Hygroscopic & {$[24,33,35]$} \\
\hline LYSO(Ce) & $(65)$ & 7.1 & $25-33$ & $<15 \%$ & $10^{3} \mathrm{~cm}^{3}$ & ${ }^{176} \mathrm{Lu}$ activity & {$[33,36,37]$} \\
\hline CLYC(Ce) & (54) & 3.31 & 20 & $4.8 \%$ & $10^{2} \mathrm{~cm}^{3}$ & $\begin{array}{l}\text { Hygroscopic, } \\
\text { internal activity }\end{array}$ & {$[38,39]$} \\
\hline
\end{tabular}

In scintillator detectors, the number of e-h pairs created by incident radiation are counted using a photomultiplier to detect the electromagnetic radiation (scintillation photons) emitted upon the radiative recombination of electrons and holes. The peak emission wavelength of the scintillation photons is dependent on the crystal composition but for desirable scintillators, it typically lies around the visible light region of the electromagnetic spectrum, enabling efficient collection by photomultipliers [24]. Since statistical fluctuations are present in the number of scintillation photons produced per unit energy deposited in the scintillator (light yield), higher light yields contribute to a higher energy resolution, as shown in Table 1 for $\mathrm{NaI}(\mathrm{Tl})$ and $\mathrm{LaBr}_{3}(\mathrm{Ce})$, with light yields of 38 and 63 photons 
per keV, respectively $[24,32,35]$. Another important property for scintillators to possess is minimal overlap between their absorption and emission spectra, such that they are transparent to the majority of scintillation photons produced. In inorganic scintillators the presence of dopant atoms creates additional states lying within the band gap of the electronic structure of the crystal, providing a means for e-h recombination that produces scintillation photons that cannot be reabsorbed and re-excite the crystal $[24,28]$. In contrast, organic scintillators (e.g., PVT) do not reabsorb the majority of e-h recombination photons due to the presence of short-lived molecular vibrational states through which electrons and holes can rapidly recombine.

Organic scintillators, which typically consist of a scintillating organic (e.g., p-terphenyl) suspended in a solid or liquid solvent (e.g., PVT)), are cheap and can be easily manufactured with large cross sectional areas $\left(\sim \mathrm{m}^{2}\right)$, giving them high geometric efficiencies [24,26]. One limitation of organic scintillators, which is a consequence of their low $\mathrm{Z}$ and densities, as shown in Table 1, is their extremely poor energy resolution. For $\gamma$ radiation, the dominant interaction process in organic scintillators is Compton scattering, meaning only a fraction of the energy of an incident $\gamma$ is deposited into the scintillator. Hence there is limited spectral information in the output detector spectrum; there are no clear characteristic photopeaks corresponding to the incident $\gamma$ depositing all its energy in the scintillator to allow for emitter identification. A solution to improve the energy resolution of plastic scintillators is to impregnate them with high $\mathrm{Z}$ number elements; one study achieved an energy resolution of $16 \%$ at $662 \mathrm{keV}$ using a $14 \mathrm{in}^{3}$ bismuth-loaded PVT plate [40], despite the unwanted effects of reduced light yield (to $6 \mathrm{ph} / \mathrm{keV}$ ) and increased scintillation photon attenuation associated with impregnating the PVT with Bi [24].

In contrast to the low energy resolution, the low $\mathrm{Z}$ make-up of organic scintillators gives them intrinsic fast neutron detection capability; in elastic scattering interactions, neutrons transfer the largest fraction of their energy when colliding with particles of the same mass [41]. To differentiate between incident neutron and $\gamma$ particles, pulse shape discrimination (PSD) can be used to compare the variation of the measured current pulse in time, which is different for neutron and $\gamma$ events because of the different means through which they interact in the scintillator [42,43]. In order for organic scintillators to detect thermal neutrons, they can be loaded with materials with high thermal neutron capture cross sections, such as ${ }^{10} \mathrm{~B}$ [42]. One liquid organic scintillator commonly used for neutron counting is EJ-309, which is commercially available with and without boron loading [31]. Despite the neutron detection capabilities of organic scintillators, the standard detectors used for neutrons are ${ }^{3} \mathrm{He}$ gas proportional counters. ${ }^{3} \mathrm{He}$ has an extremely high thermal neutron capture cross section at 5330 barns and can be adapted to detect fast neutrons by surrounding the gas chamber in a moderating material such as polyethylene to thermalise the neutrons $[5,41]$. A moderated ${ }^{3} \mathrm{He}$ detector has high detection efficiency $(76 \%$ detection efficiency for a $0.025 \mathrm{eV}$ neutron travelling through $2 \mathrm{~cm}$ of ${ }^{3} \mathrm{He}$ at $5 \mathrm{~atm}$ ) across a range of neutron energies owing to the presence of the moderator, as well as good gamma rejection properties [24,41].

There has been a considerable amount of research undertaken into inorganic scintillators since the discovery of $\mathrm{NaI}(\mathrm{Tl})$ in 1948, particularly at the start of the 21st century, thanks to the concerns around national security previously alluded to in Section 1 [41]. As shown in Table 1, inorganic crystals typically have much higher (effective) $\mathrm{Z}$ and densities compared to organic scintillators; affording them greater intrinsic efficiency and energy resolution (as high as $2.7 \%$ at $662 \mathrm{keV}$ for $\gamma \mathrm{s}$ in $\mathrm{LaBr}_{3}(\mathrm{Ce})$ ). Their main disadvantage is the fact that pure, single crystals are required to prevent charge trapping, the trapping of e-h pairs that prevents or delays their recombination and inhibits the detection of radiation incident on the detector [27,41]. Growing large, single crystals is difficult, time consuming and complex, explaining the difference between the available sizes of inorganic and PVT scintillators (shown in Table 1) and justifying their considerably greater cost.

One emerging inorganic elpasolite scintillator is Caesium Lithium Yttrium Chloride (Ce) (CLYC), which has a relatively high energy resolution for $\gamma$ radiation $(4.8 \%$ at 
$662 \mathrm{keV}$ [44]) and intrinsic fast neutron sensitivity due to the the neutron capture cross section of ${ }^{35} \mathrm{Cl}(3.6$ barns at $1 \mathrm{MeV})[45,46]$. In addition, by controlling the ratio of the isotopes ${ }^{6} \mathrm{Li}$ and ${ }^{7} \mathrm{Li}$ in CLYC, the sensitivity to thermal neutrons can be adjusted, as ${ }^{6} \mathrm{Li}$ has a high thermal neutron capture cross Section (940 barns) [24,39]. Used with pulse shape discrimination (PSD), CLYC represents a "dual mode" scintillator, capable of both gamma spectroscopy and neutron detection (across a range of neutron energies) [47]. Like other inorganic crystals, the available sizes of CLYC crystals are limited. However, in recent work, a plastic composite comprising CLYC (or another inorganic scintillator) encased in a plastic scintillator was proposed with comparable $\gamma$ efficiency to $\mathrm{NaI}(\mathrm{Tl})$, dual mode capability and the potential to be scaled to larger sizes [43].

Whatever the scintillator material selection, light collection by a photomultiplier can be maximised by matching the peak emission wavelength of the scintillator with the peak wavelength efficiency of the specific photomultiplier, as well as choosing an appropriate arrangement of photomultipliers, reflecting surfaces and light shielding around the scintillator. An illustrative diagram of a scintillator crystal and a photomultiplier is shown in Figure 3; the purpose of a photomultiplier is to convert photons into a measurable electrical signal. One type of photomultiplier, the photomultiplier tube, or PMT, has been used ubiquitously throughout scientific studies since its inception in the 1930s [48].

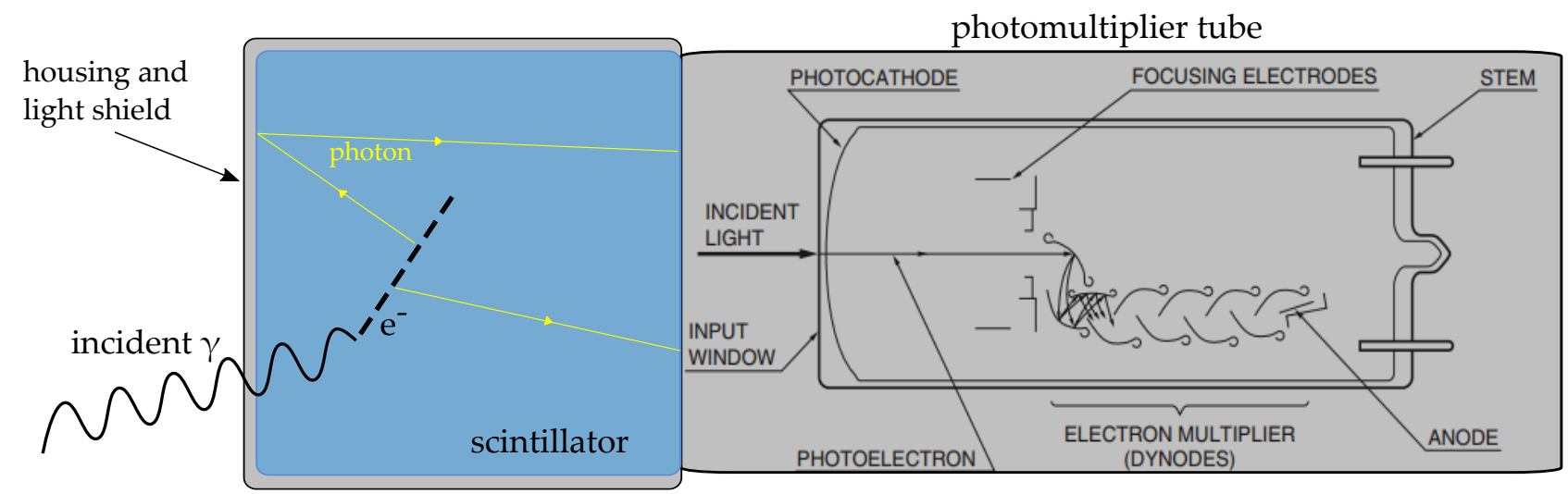

Figure 3. Illustrative diagram of scintillator detector and photomultiplier tube (PMT) (not to scale) [24]. PMT diagram reprinted, with permission, from [49]. Courtesy of Hamamatsu Photonics K.K.

Scintillation photons incident onto the photocathode of the PMT transfer energy to electrons by the photoelectric effect, causing photoelectrons to be ejected from the photocathode; the number of photoelectrons emitted per incident photon is defined as the quantum efficiency (for common photocathodes the maximum is $20-30 \%$ ) [24,27]. Once a photoelectron has been ejected from the photocathode, it is accelerated by an electric field through a vacuum chamber that contains successive multiplying electrodes so that a measurable signal reaches the anode, where it is registered as a current pulse that is proportional to the number of photoelectrons created at the photocathode, and therefore the number of scintillation photons $[24,50]$.

Despite their capability, PMTs have become increasingly replaced by semiconductor photomultipliers in scintillator detector applications [51,52]. One type of semiconductor photomultiplier is the silicon photomultiplier (SiPM), which consists of an array of microcells, each of which is a photodiode operated in Geiger avalanche mode, called a single photon avalanche diode (SPAD) [50,51]. When scintillation photons are incident on an avalanche photodiode, an e-h pair is created in the semiconductor that, in the presence of an electric field, causes an avalanche of ionisation, constituting a current pulse; the combined current pulse of the entire array of microcell photodiodes is proportional to the number of incident scintillation photons [51]. The main benefits of SiPMs, compared to PMTs, are their ruggedness, lower cost, lack of sensitivity to magnetic fields and a smaller form factor [50]. Furthermore, through using large area avalanche photodiodes 
improved energy resolution can be achieved, with reductions to $4.9 \%$ FWHM at $662 \mathrm{keV}$ reported for CsI(Tl) scintillators $[53,54]$. For both SiPMs and PMTs the wavelength of peak sensitivity is similar at 450 and $420 \mathrm{~nm}$, respectively; alongside the quantum efficiencies at these wavelengths, hence, scintillator materials with scintillation photons in a similar wavelength range should be selected [50,55].

All of the detector materials, ranging from organic PVT to novel inorganic elpasolites, represent potential candidate materials for use in shipping container screening applications. In such screening applications, efficiency is perhaps the most important detector property, as by determining the fraction of emitted radiation that is registered by a detector it affects the likelihood of detecting a concealed source. This is especially important in shipping container screening where there are large stand-off distances between a detector and any source within a container; for a detector positioned in contact with one side of a shipping container such that it measures across the shortest dimension of the container (its width), a source in the detector line of sight can be anywhere between 0 and $2.43 \mathrm{~m}$ away. It is crucial, therefore, for the geometric efficiency of detectors to be maximised at such stand-off distances and, as a consequence, low cost is another desirable requirement for detector materials to be used in shipping container screening applications, due to the relationship between the geometric efficiency and the amount of detector material required. For an enhanced shipping container screening capability, high energy resolution also is essential, as it allows for the isotopic characterisation of radioactive material within a shipping container, and therefore informs decisions on whether radioactive material in cargo constitutes a legitimate threat or is a nuisance source, such as NORMs.

\section{Radiation Detection Methods for Use in Non-Intrusive Shipping Container Screening}

\subsection{Requirements of Non-Intrusive Shipping Container Screening Systems}

An ideal screening system identifies every source and returns no false alarms. In practice, this is an impossible scenario due to false alarms from fluctuations in background and electrical noise, nuisance sources (typically, NORMs such as ${ }^{40} \mathrm{~K}$ in fertiliser) and medical material (e.g., ${ }^{99 m} \mathrm{Tc}$ ), therefore screening systems are designed to operate within acceptable true detection and false-positive alarm rates. In the case of the radiation screening of shipping containers within a busy seaport environment, this amounts to a trade-off of operational resources and transportation delays, versus complete detection [16].

As formerly alluded to, alarms can be split into four groups: true positives, false positives, true negatives, and false negatives [56]. A receiver operating characteristic (ROC) curve is a plot of the false-positive rate against the true positive rate, with the true positive rate (TPR, or sensitivity) defined as

$$
\mathrm{TPR}=\frac{T_{p}}{T}=\frac{T_{p}}{T_{p}+F_{n}}
$$

where $T$ is the number of positive events, $T_{p}$ is the number of true positives, and $F_{n}$ the number of false negatives. Similarly, the false-positive rate (FPR, or fall-out) can be defined as

$$
\mathrm{FPR}=\frac{F_{p}}{F}=\frac{F_{p}}{F_{p}+T_{n}}
$$

where $F$ is the number of negative events, $F_{p}$ the number of false positives, and $T_{n}$ the number of true negatives.

Figure 4 shows potential ROC curves for various scenarios. In the case of ideal performance, TPR is observed to be unity (all positive events correctly classified) and FPR is zero (no negative events incorrectly identified as positive) as shown by the pink star. The blue line shows the expected performance of the screening system if it were to randomly guess if a source were present, with the orange and green lines showing increasing TPR at lower FPR values, indicating an improvement in performance [56]. Such 
ROC curves are useful as they facilitate the comparison of false alarm probabilities of different screening systems at set detection efficiencies (or vice versa), as well as providing a means of comparing the performance of screening systems to recognised standards set by organisations such as the International Atomic Energy Agency (IAEA) and American National Standards Institute (ANSI).

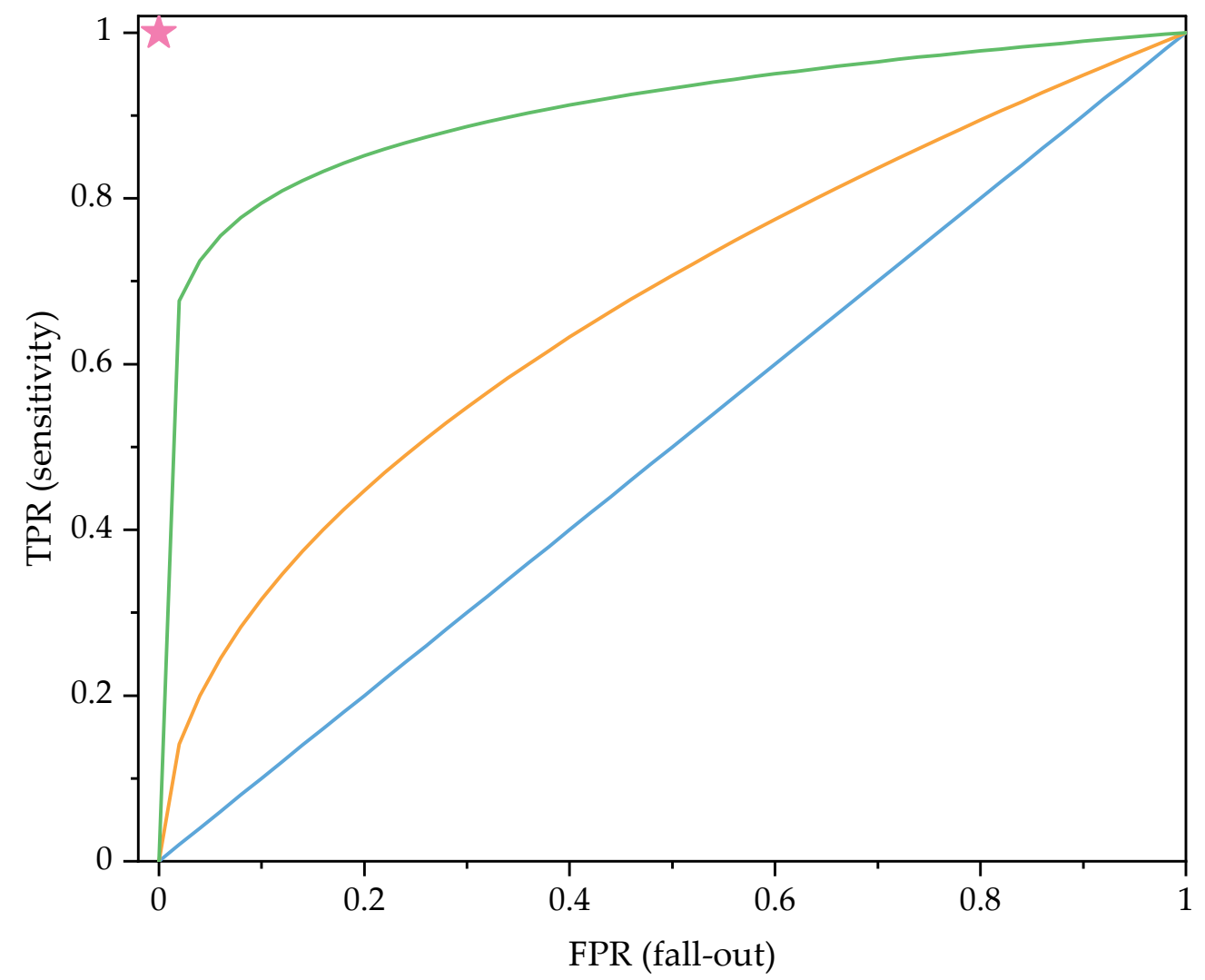

Figure 4. Example receiver operating characteristic (ROC) curves. The pink star indicates the ideal performance of a screening system whereas the green and orange lines represent successively worse performance. The blue line is the expected performance if the screening system randomly guessed.

Through the framework of ROC curves, the performance of different radioactive screening systems for shipping containers can be compared. Table 2 shows both essential and desirable requirements of such screening systems, the metrics they are evaluated with alongside brief notes on how they may be met. Requirements such as the initial cost and ongoing maintenance costs of a screening system determine its affordability, which represents an important factor for a system that needs to be implemented at seaports nationwide. The influence of cost is highlighted by the choice of detector that is most widely used in practice-namely PVT-based detectors. Further operational requirements include tolerance to the harsh environments associated with the variable weather conditions at seaports and deployability at the smallest seaports (achieved via mobile detector units), which improves the deterrent quality of a nationwide screening capability $[1,12]$. 
Table 2. Requirements of shipping container screening systems for detecting illicit radioactive or nuclear materials.

\begin{tabular}{|c|c|c|}
\hline Requirement & Metric & Methods to Meet Requirement \\
\hline High sensitivity & TPR & $\begin{array}{l}\text { Use high-efficiency radiation detectors; maximise the duration for } \\
\text { inspection to take place; position detectors close to shipping containers. }\end{array}$ \\
\hline Minimise false alarms & FPR & $\begin{array}{l}\text { Use high energy resolution detectors to identify threat sources; } \\
\text { use advanced algorithms to process data from low energy resolution } \\
\text { detectors; continuously monitor background radiation. }\end{array}$ \\
\hline High throughput & $\begin{array}{l}\text { Delays } \\
\text { caused }\end{array}$ & $\begin{array}{l}\text { Minimise duration of inspection; inspect shipping containers in transit; } \\
\text { minimise false alarms. }\end{array}$ \\
\hline Affordability & $£$ & $\begin{array}{l}\text { Choice of detector material; number of screening systems required per } \\
\text { seaport; minimise manual operational requirements. }\end{array}$ \\
\hline Mobility & & Mount the screening system on a vehicle. \\
\hline Durability & & Use weather resistant materials in detector housing. \\
\hline Isotopic identification & $\begin{array}{l}\text { Detector } \\
\text { resolution }\end{array}$ & $\begin{array}{l}\text { Use high-resolution detector materials; use high-efficiency detectors; } \\
\text { implement peak identification algorithms. }\end{array}$ \\
\hline Source localisation & $\begin{array}{l}\text { Localisation } \\
\text { error }\end{array}$ & $\begin{array}{l}\text { Use a distributed array of detectors; use advanced data processing } \\
\text { techniques to estimate source location; choose an appropriate } \\
\text { arrangement and number of detectors. }\end{array}$ \\
\hline
\end{tabular}

Since illicit sources may be intentionally shielded and their potential end uses could be catastrophic, high sensitivity is an essential requirement for screening systems, which can be achieved by using efficient radiation detectors; either geometrically efficient organic scintillators or arrays of smaller, intrinsically efficient, inorganic crystal scintillators [13,26]. On the other hand, high sensitivity must not come at the cost of high fall-out, as each false alarm must be verified, and therefore decreases the throughput of the screening system; taking up operational resources. An effective way to minimise nuisance alarms is to identify the specific isotopes based on their characteristic spectra and decide whether the isotopes constitute a threat, for example, the $414 \mathrm{keV} \gamma$ peak from ${ }^{239} \mathrm{Pu}$ decay is an indication of the presence of $\mathrm{WGPu}$, whereas the $1460 \mathrm{keV} \gamma$ peak from ${ }^{40} \mathrm{~K}$ decay is an indicator for NORMs [16,57]. Methods have been developed for differentiating between certain types of radioactivity (e.g., NORMs and SNMs) using low energy resolution detectors (which are subsequently discussed in Section 4.1), however, high energy resolution radiation detectors provide the best means of identifying specific isotopes as they produce well-defined spectra from which characteristic peaks can be easily resolved.

Beyond the basic requirements of efficient detection and minimal impact on shipping container throughput, future capabilities of screening systems should include estimation of the location of a source within a shipping container, full isotopic classification of the source and estimation of the activity of the source. To perform this, complex data processing algorithms could be used, relying on models of the expected detector response to various gamma or neutron-emitting sources at differing locations. Ultimately, the capabilities of future screening systems should serve to minimise the manual operational requirements of shipping container screening, as well as effectively protect nations from the potential consequences of the unchecked transport of radioactive materials.

\subsection{Currently Deployed Non-Intrusive Shipping Container Screening Systems}

Current shipping container screening systems operate a two-stage process. In the initial screening stage, the container is driven at restricted speeds (up to $8.3 \mathrm{~ms}^{-1}$ ) through an RPM with a large cross section, surrounding an individual traffic lane, as shown in Figure $5[13,58,59]$. Combined with the low transit speeds that increase the duration of the inspection, the sensitivities of primary stage RPMs are typically maximised through the use of large cross section PVT or polystyrene scintillator panels (with high geometric efficiency) for $\gamma$ detection and separate neutron detectors-typically ${ }^{3} \mathrm{He}$ gas detectors $[26,59]$. The benefit of using plastic scintillator RPMs is their low production cost, enabling multiple primary RPMs to be deployed at a checkpoint so a high throughput of shipping containers 
can be maintained and the resultant impact on their transportation minimised. However, the poor energy resolution of these plastic scintillators necessitates a second screening stage to investigate any alarms that are generated during the first stage, and if needed, identify and locate sources concealed within a container. In the secondary phase, suspect shipping containers are diverted from the traffic flow to be inspected with high energy resolution detectors; typically, this is performed manually using handheld radioisotope identification devices or remotely using a single RPM with spectroscopic capabilities. However, in either case, the detectors are likely to utilise $\mathrm{NaI}(\mathrm{Tl})$ or $\mathrm{LaBr}_{3}(\mathrm{Ce})$ scintillators $[13,60]$.

To determine which shipping containers require secondary inspection, a suitable algorithm to process the measurements made by an RPM during the primary phase must be selected. Currently, the "counts above threshold" alarm criteria is most widely used in RPM-based screening systems, which is a simple test statistic that compares the number of radiation detection events (counts) measured by an RPM in the presence of a shipping container to the number of counts measured from background radiation alone [14]. The test statistic, $T$, is defined as

$$
T=\frac{\lambda-\lambda_{b}}{\sqrt{\lambda_{b}}}
$$

where $\lambda$ is the counts measured by an RPM, and $\lambda_{b}$ is the background counts [14,61]. This test statistic, $T$, represents the number of counts above the background level as a multiple of the standard deviation of the (Poisson/Gaussian) background distribution. During the transit of a shipping container through an RPM, a sequence of fixed interval measurements are taken and $T$ is resultantly calculated for subsequent measurements [14]. If $T$, for any measurement, exceeds a threshold value set using the desired TPR and FPR of the primary screening system, then the shipping container is flagged for secondary inspection.

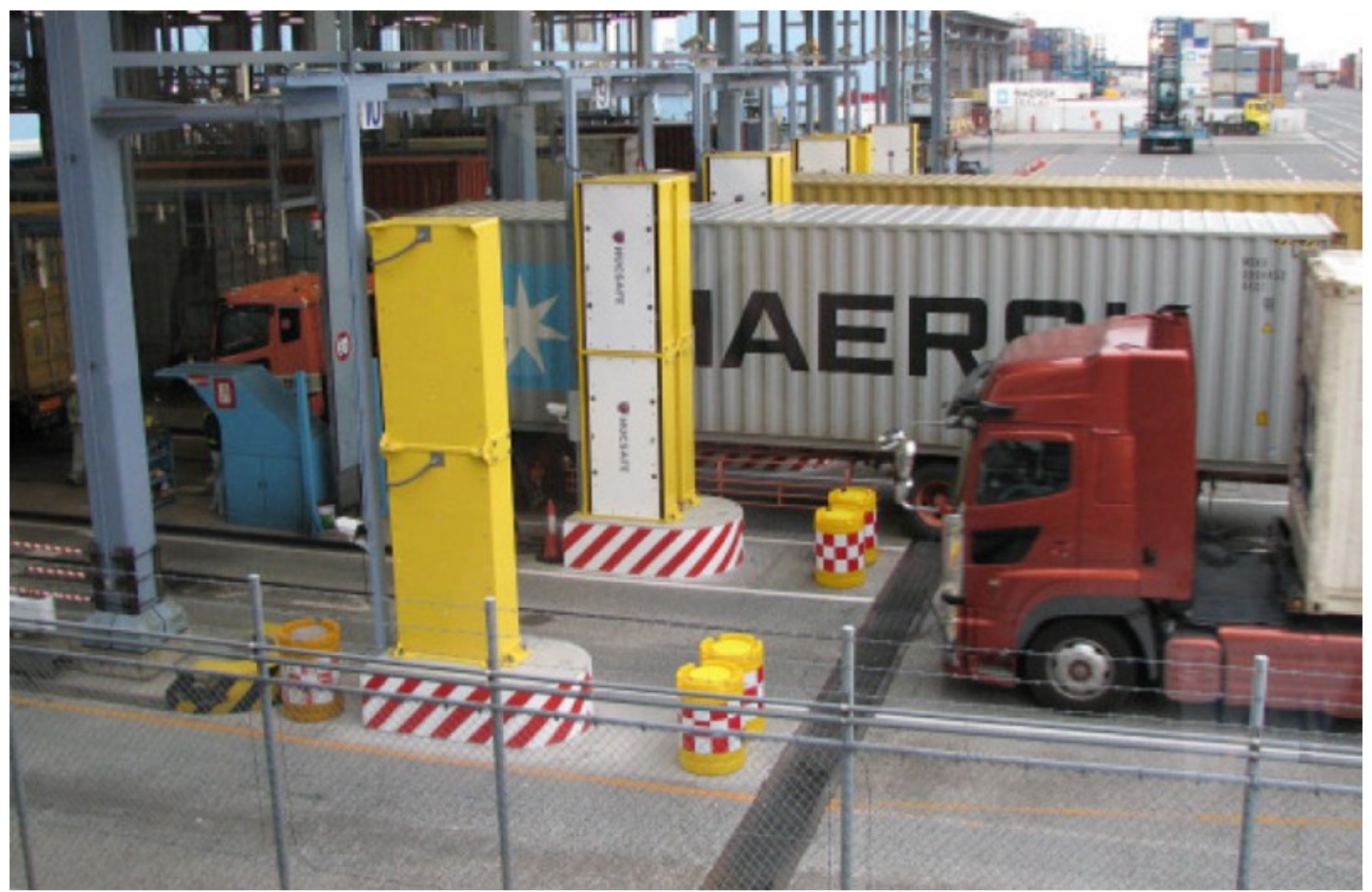

Figure 5. Shipping containers driven on flatbed trucks through vertical radiation portal monitors (white panels in yellow housing). Reprinted, with permission, from [59]. (C) American Science \& Engineering, Inc., An OSI Systems Company.

The result in Equation (4) represents one of the simplest means of determining what should trigger an alarm and, when used alone, offers no means of differentiating between legitimate and nuisance alarms. Accordingly, various methods exist for improving the 
performance of this algorithm, such as calculating $T$ for counts in specific energy channels (or larger energy bands) to identify increases in counts at energies characteristic of certain isotopes (e.g., around $662 \mathrm{keV}$ for ${ }^{137} \mathrm{Cs}$ ). Alternatively, through obtaining repeat measurements as a shipping container moves through an RPM, NORM materials that are typically characterised by large amounts of low-activity source distributed homogeneously throughout the whole container volume can be differentiated from smaller, point-like sources of higher activity by considering the variation of the counts over the time it takes for the container to be driven through the RPM [16]. A more detailed overview of the methods that can be used to improve the performance of primary RPM screening systems is given in Section 4 .

One effect that must be accounted for with RPMs is baseline suppression, which occurs when detectors are partially shielded from background radiation by the presence of a shipping container passing in front of the detector $[62,63]$. The extent of this effect is dependent on shipping container shape and composition and is shown to vary across detector sites even with the same detection system [62]. In Equation (4), if $\lambda$ is measured with a suppressed rate of background counts, then the test statistic $(T)$ will be lower than its true value unless $\lambda_{b}$ is corrected to be equal to the suppressed background count rate. This correction can be made by quantifying the background count rate during the measurement of the shipping container in the RPM or via modelling the suppression effect and correcting the background rate measured with no shipping container present.

\subsection{Modelling Screening Systems}

Establishing a model for the anticipated number of counts in detectors used in shipping container screening allows for the performance to be understood and, when more complicated data processing algorithms are used, is fundamental to their operation. The models reviewed in this work rely on the assumption that the underlying distributions of the source and background counts are Poissonian, which is a standard assumption for $\gamma$ and neutron radiation detection applications and holds in the case that the detection time is small when compared to the source half-life (as the probability of a Poisson event is small and constant) [24,64-66]. The probability of observing $x$ detector counts, given by the Poisson distribution is;

$$
\mathbb{P}(x \mid \lambda)=\frac{\exp (-\lambda) \lambda^{x}}{x !}
$$

where $\lambda$ is the mean number of counts characterising a source, or the probability of detection multiplied by the number of events [24]. This parameter can also be split into $\lambda_{b}+\lambda_{s}$ in order to differentiate between the background $\left(\lambda_{b}\right)$ and source $\left(\lambda_{s}\right)$ counts and based on the description in Section 2, namely Equation (1), can be expressed as

$$
\lambda=\lambda_{b}+\lambda_{s}=\frac{\lambda_{0}}{d^{2}} \exp \left(-\int_{r} \mu_{r} d r\right)+\lambda_{b}
$$

where $\lambda_{0}$ is the source counts measured by the detector $1 \mathrm{~m}$ from the source, $d$ represents the distance from the source to the detector, $\mu_{r}$ is the linear attenuation coefficient at a position $r$ along the integral path between the source and the detector, and $\lambda_{b}$ is the mean background counts at the detector [67]. For simplicity, both the detector cross section and $\frac{1}{4 \pi}$ factor are included in the $\lambda_{0}$ term to account for the solid angle of the detector face and a build up factor for radiation scattering into the path of the detector is omitted.

Unlike smaller consignments of goods, the considerably larger shipping containers, up to 40 feet in length, are not typically filled homogeneously, or with a single materialcontaining combinations of both air gaps and assorted materials/cargo. Without a complete description of the container contents, the complex attenuation profile cannot be perfectly described and for this reason, alongside the inherent complexity that it introduces into the numerical calculations, the exponential term in Equation (6) accounting for material attenuation is often simplified or completely ignored. One simplification, to address this problem, assumes homogeneous cargo within the container, so the attenuation depends 
purely on the distance between the source and detector, whereas a more complex method would attempt to quantify the shielding from different materials within the cargo and their placement relative to the source and detector by means such as X-ray radiography or assessment of the containers inventory [68].

Another important use of models in the development of screening systems is to generate data that novel processing algorithms can be validated with; there exists two main methods of modelling source and background count distributions. Using an appropriate mathematical model, such as Equation (5), counts from different sources and background (characterised by $\lambda$ ) can be generated [61]. Alternatively, Monte Carlo simulations of source-detector scenarios can be performed with computational models using software such as the GEANT4 package developed at CERN, which can output the energy (and total counts) deposited in detectors from user defined sources $[69,70]$. These methods of modeling screening systems provide a means for testing novel data processing algorithms (and detector materials in the case of Monte Carlo-based computational models) without requiring experimental data that can be be significantly more costly to obtain. Obviously, experimentally derived data with physical sources and detectors represents the most useful method of obtaining this necessary validation data as it reflects the "ground truth" of the scenario [71]. In support of testing and validating research into such algorithm-based detection systems, the US Domestic Nuclear Detection Office's Intelligence Radiation Sensors Systems (IRSS) program [71] has made experimental datasets publicly available which have been used extensively to underpin the various works discussed in this review. These datasets comprise empirical measurements for different radioactive sources, background environments, detector configurations and source trajectories.

\section{Advancements in Radiation Portal Monitor Screening Processes}

Analysing the specific data processing algorithms employed in commercially available RPMs to raise alarms during the primary screening process is difficult, as industrial vendors are in direct competition to attain the best inspection performance and, from a deterrent standpoint, it is not advisable for nations to make the exact specifications of their screening systems publicly available. Consequently, the exact technological nature and level of infrastructure deployment of these methodologies cannot be truly known - with some approaches having reached full-scale realisation while others may still remain under active development.

\subsection{Source Characterisation Based on Energy Considerations}

Owing to the wide implementation of RPMs, many methods for discriminating between nuisance alarms and legitimate threat sources, using the energy distribution of the counts measured by a radiation detector, have been developed. For high-resolution radiation detectors such as $\mathrm{NaI}(\mathrm{Tl})$ or $\mathrm{LaBr}_{3}(\mathrm{Ce})$, this simply involves photopeak identification; a Gaussian curve can be fitted to a photopeak to determine the energy centroid of the peak, which can be compared to values in a look-up table to identify the source isotope [27]. In contrast, as formerly alluded to in Section 2.2, the energy spectrum of plastic scintillators is dominated by the low energy Compton continuum, so radionuclides are not identifiable using characteristic photopeaks and more sophisticated methods must be used.

One such method, energy windowing (EW), utilises algorithms to discriminate between NORMs, SNMs and other radionuclides using prior knowledge of the expected spectrum shapes of the different source types $[15,72,73]$. NORM cargo has a similarly shaped energy spectrum to background radiation, whereas SNMs have elevated counts in low energy channels. Therefore, by taking ratios of the number of counts in different regions of the spectra, EW allows for NORMs/background sources and SNMs to be differentiated from one another [15]. Used in conjunction with the counts above threshold method, EW algorithms provide a means of minimising the number of false alarms and, in addition, are not sensitive to the effects of background suppression as they rely on ratios of counts as opposed to the gross number of counts [15,72]. 
Depending on the specifics of an EW algorithm, the energy spectrum is split into any number of energy windows based on specific spectral regions of interest, which, for a specific isotope typically includes the low-energy Compton edge [15]. To identify SNMs and material that may be used in a RDD, one EW method proposed in [72] uses energy window regions based on calibration measurements taken to find the regions of interest for HEU, WGPu, ${ }^{137} \mathrm{Cs}$, DU and ${ }^{40} \mathrm{~K}$ sources alongside a "noise" window [72]. By injecting modelled source profiles into real RPM measurements of different cargo types, such as cargo containing ${ }^{40} \mathrm{~K}$, granite or no radioactive material, in tests this EW algorithm achieved $100 \%$ sensitivity for sufficiently high-activity injections of HEU, WGPu, ${ }^{137} \mathrm{Cs}$ and ${ }^{40} \mathrm{~K}$ sources, except when HEU and WGPu profiles were injected into the measurements of granite bearing cargo, where the radioactivity of the granite limits the ability of the algorithm to correctly identify such sources [72].

An alternative approach to EW for identifying specific radionuclides is to analyse the measured spectra by comparison with template spectra from a library of known sources using methods such as a least squares fitting (LSF) comparison, after manipulating the spectra to emphasise features and suppress noise [74]. One variation of this is a spectral angle mapping (SAM) method, which quantifies the similarity between measured and reference spectra by finding the angle between their cumulative count distributions in Fourier space, which has the effect of comparing the shape of the measured and reference cumulative count distributions-rather than absolute values as would be compared by a least squares method [75]. Using an organic scintillator (EJ-309) detector configuration, the SAM method was shown to identify a range of radioisotopes including ${ }^{137} \mathrm{Cs},{ }^{241} \mathrm{Am}$ and ${ }^{99 \mathrm{~m}} \mathrm{Tc}$, moving through a pedestrian RPM at speeds of $2.2 \mathrm{~ms}^{-1}$ with activities less than or similar to ANSI standards (ANSI N42.35, 2006), with a total sensitivity of 0.92 [75]. A drawback to LSF and SAM methods is that their efficient operation relies on large libraries of measurements for the correct identification of isotopes. However, they have been demonstrated to be capable of introducing a radioisotope identification ability to plastic scintillator-based RPM screening systems.

A simpler method identifies isotopes by finding peaks in the energy/channel weighted spectra, which occur at the point of the Compton edge for isotopes with significant Compton continuums, such as ${ }^{137} \mathrm{Cs}$, hence characteristic photopeak energies can be calculated using the Compton scattering equation [76]. In isotopes where the spectra contains multiple low-energy $\gamma \mathrm{s}$ and there exists no readily discernable Compton edge, such is the case with ${ }^{238} \mathrm{U}$, there still exists a characteristic peak in the energy weighted spectrum which can be determined experimentally. This method was shown to identify sources $\left({ }^{238} \mathrm{U},{ }^{226} \mathrm{Ra},{ }^{137} \mathrm{Cs}\right.$, ${ }^{60} \mathrm{Co}$ ) moving through a PVT RPM at $2.8 \mathrm{~ms}^{-1}$, at activities specified within standards (ANSI N42.38, 2006) for ${ }^{137} \mathrm{Cs}$ and ${ }^{60} \mathrm{Co}[77]$.

Table 3 compares the performance of the EW, LSF, SAM and energy weighted methods for radioisotope identification. Clearly, all of these methods represent a means of improving the ability of a primary, plastic scintillator-based, RPM to discriminate between different types of radioactive or nuclear material. By integrating such energy-based algorithms into existing RPM systems, the primary screening phase can invoke informed alarm decisions, by correctly identifying threat sources, such as WGPu and ${ }^{137} \mathrm{Cs}$. Used in combination with counts above threshold alarm criteria, these methods enable existing plastic scintillatorbased RPMs to meet the performance requirements, such those set by ANSI and the IAEA. 
Table 3. Results of energy-based methods for radioisotope identification of sources moving through plastic scintillator-based RPMs. Identification probability (IP) is the probability of the correct radioisotope identification.

\begin{tabular}{|c|c|c|c|c|c|}
\hline Method & Refs. & Source & Source Speed & IP & Notes \\
\hline EW & {$[15,72,73]$} & $\begin{array}{l}555 \mathrm{cps}{ }^{137} \mathrm{Cs} \\
1110 \mathrm{cps} \text { WGPu }\end{array}$ & $\begin{array}{l}- \\
-\end{array}$ & $\begin{array}{l}1.0 \\
1.0\end{array}$ & $\begin{array}{l}\text { Theoretical source profiles injected } \\
\text { into existing non-alarming vehicle } \\
\text { RPM data. }\end{array}$ \\
\hline LSF & [74] & $\begin{array}{c}370 \mathrm{kBq}{ }^{137} \mathrm{Cs} \\
259 \mathrm{kBq}{ }^{60} \mathrm{Co} \\
6.6 \mathrm{~g} \mathrm{WGPu}\left(93 \%{ }^{239} \mathrm{Pu}\right) \\
\end{array}$ & $\begin{array}{l}2.2 \mathrm{~ms}^{-1} \\
2.2 \mathrm{~ms}^{-1} \\
1.2 \mathrm{~ms}^{-1} \\
\end{array}$ & $\begin{array}{c}0.83 \\
0.67 \\
0\end{array}$ & $\begin{array}{l}\text { Single panel pedestrian RPM; LSF } \\
\text { to cumulative count distribution; } \\
\text { EJ-309 organic scintillator. }\end{array}$ \\
\hline SAM & [75] & $\begin{array}{c}370 \mathrm{kBq}{ }^{137} \mathrm{Cs} \\
\text { 6.6 g WGPu }\left(93 \%{ }^{239} \mathrm{Pu}\right)\end{array}$ & $\begin{array}{l}2.2 \mathrm{~ms}^{-1} \\
2.2 \mathrm{~ms}^{-1}\end{array}$ & $\begin{array}{l}1.00 \\
0.93\end{array}$ & $\begin{array}{l}\text { Single panel pedestrian RPM; } \\
\text { EJ-309 organic scintillator. }\end{array}$ \\
\hline $\begin{array}{c}\text { Energy } \\
\text { weighted }\end{array}$ & {$[76,77]$} & $\begin{array}{l}340 \mathrm{kBq}{ }^{137} \mathrm{Cs} \\
237 \mathrm{kBq}{ }^{60} \mathrm{Co}\end{array}$ & $\begin{array}{l}2.8 \mathrm{~ms}^{-1} \\
2.8 \mathrm{~ms}^{-1}\end{array}$ & $\begin{array}{l}0.6 \\
0.8\end{array}$ & PVT-based vehicle RPM. \\
\hline
\end{tabular}

\subsection{Data Processing Algorithms for Improved Sensitivity}

A range of data processing algorithms, which operate on the set of sequential measurements taken by a single RPM as a shipping container passes through it, have been identified as possible replacements for the aforementioned counts above threshold method.

The first method, a sequential probability ratio test (SPRT), is an established method for comparing a null hypothesis against another hypothesis; in the case of radiation detection, the null hypothesis is that the measured counts originate from background radiation-with the alternative hypothesis that the measured counts are the combined effects of background and another radiation source $[65,78,79]$. For the sequence of measurements taken by an RPM, the alternative hypothesis is confirmed by the sum of the log likelihood ratios, calculated for each measurement, exceeding a threshold, where the log likelihood ratio, $\Lambda$, is given by;

$$
\Lambda=\ell\left(H_{1} \mid x\right)-\ell\left(H_{0} \mid x\right)=\log \left(\frac{\mathcal{L}\left(H_{1} \mid x\right)}{\mathcal{L}\left(H_{0} \mid x\right)}\right),
$$

where $\mathcal{L}\left(H_{1} \mid x\right)$ is the likelihood of the alternative hypothesis, $H_{1}$, and $\mathcal{L}\left(H_{0} \mid x\right)$ is the likelihood of the null hypothesis, $H_{0}$, given the measurement $x$ (counts) $[61,65]$. The test statistic, $T_{k}$, after the $k$ th measurement, is thus given by $T_{k}=T_{k-1}+\Lambda_{k}$.

The SPRT methodology relies on assumptions of the likelihoods of the individual hypotheses, for example, $\mathcal{L}\left(H_{0} \mid x\right)$ defined using Equations (5) and (6) yields the result;

$$
\mathbb{P}\left(x_{i} \mid \lambda_{b}\right)=\frac{\exp \left(-\lambda_{b}\right)\left(\lambda_{b}\right)^{x_{i}}}{x_{i} !}
$$

for the null hypothesis, where $x_{i}$ is the counts in the $i$ th measurement [65]. After this derivation, suitable values for $\lambda_{b}$ and $\lambda_{s}$ (defining the null and alternative hypotheses respectively) are chosen, where $\lambda_{s}$ is defined relative to $\lambda_{b}$, based the on desired FPR and TPR. Benefits of SPRT over the counts above threshold method, specifically over the single-interval test (SIT) where the test statistic is equivalent to the sum of the counts above threshold statistic (Equation (4)) over the RPM measurement sequence, are the ability to conclusively determine if the measured counts are consistent with background and more quickly invoke alarm decisions [65].

Another method for processing RPM measurements is the matched filter (MF) algorithm, which identifies the correlation between a theoretical count profile of a source moving through an RPM and the measured count profile $[66,80,81]$. If the correlation is above a predefined threshold value, then an alarm is raised. This method requires the velocity of the source to be accurately known and assumes the emitter is a point source such that the temporal profile of the count rate measured by the RPM is bell shaped and can be mathematically approximated $[66,80]$. This theoretical count profile is normalised 
(retaining the profile shape) and its correlation with the measured count profile is found; if a source is present then a correlation is to be expected.

A similar method to MF is estimated matched filtering (EMF). Where, instead of finding the correlation between measurements and pre-calculated theoretical source profiles, this method fits the measurement data with a model of an unknown source moving through the RPM — specifically, a generalised line source [66]. The fit parameters, including background counts, source counts, source position and source length, are determined and can be used as the test statistic to decide whether a shipping container requires subsequent inspection and investigation.

One further technique for use with a sequence of RPM measurements is that of the exponentially weighted moving average (EWMA), which assigns higher weightings to more recently obtained RPM measurements, and is expressed as

$$
T_{k}=(1-\alpha) T_{k-1}+\alpha Y
$$

where $\alpha$ is a constant that controls the iterative weighting decrease per new measurement, and $Y$ is a value derived from the measurements, for example, the counts or the log likelihood ratio $[61,66,82]$. In the latter case, the test statistic is similar to that used in SPRT, but with weighted log likelihood contributions. The benefit of using EWMA, as opposed to SPRT, is that by exponentially weighting subsequent measurements, the value of the test statistic is more sensitive to changes in the log likelihood ratio (or counts), which can facilitate faster event detection and subsequent alarming.

The authors of [66] compared the performance of the RPM data processing algorithms SPRT, MF, EMF, EWMA and SIT, alongside a maximum count rate test (where the test statistic is equivalent to the maximum value of the counts above threshold statistic (Equation (4)) in the RPM measurement sequence), performed on mathematically modelled neutron sources. Their model used Poisson distributed neutron background and source count data to assess the TPR of each algorithm at a required FPR of 0.001 [66]. The result of their work is shown in Figure 6, where the TPR, or detection probability (DP), for three different source strengths is averaged over twenty-four modelled scenarios; (6) source velocities, (2) measurement intervals and (2) background count periods.

The plot of Figure 6 demonstrates that the more computationally intensive methods such as EMF, SPRT, EWMA (with $\alpha=0.3$ ), outperformed the matched filter and standard counts above threshold-based methods (MCR and SIT), although for all the methods the TPR for weak sources $\left(<5 \lambda_{b}\right)$ is low $(<60 \%)$. The EMF approach represents the best performing algorithm, with both SPRT/EWMA performing equally well, but only marginally better than MCR. In addition, [66] determined that detection probabilities were found to be greater when longer background estimate measurements were used [66]. In separate work, carried out by [61], using mathematically modeled, Poisson distributed, background and source $\gamma$ counts in RPMs, EWMA was shown to outperform SPRT, with both shown to outperform the counts above threshold method in TPR, for an FPR of 0.0001 [61].

These results suggest that for RPM applications, alternative methods to the counts above threshold data processing algorithm exist with superior detection capability. This raises the question of why these superior methods haven't been implemented in practice. One reason for this may be that the increase in detection probability/decrease in false alarm rate isn't sufficient enough to warrant the increased complexity and associated computational cost of these superior algorithms. In addition, the counts above threshold method is often used in unison with the previously discussed energy algorithms, such as EW, which facilitates the identification of nuisance alarm events and improves the overall performance of the primary screening phase [15]. 


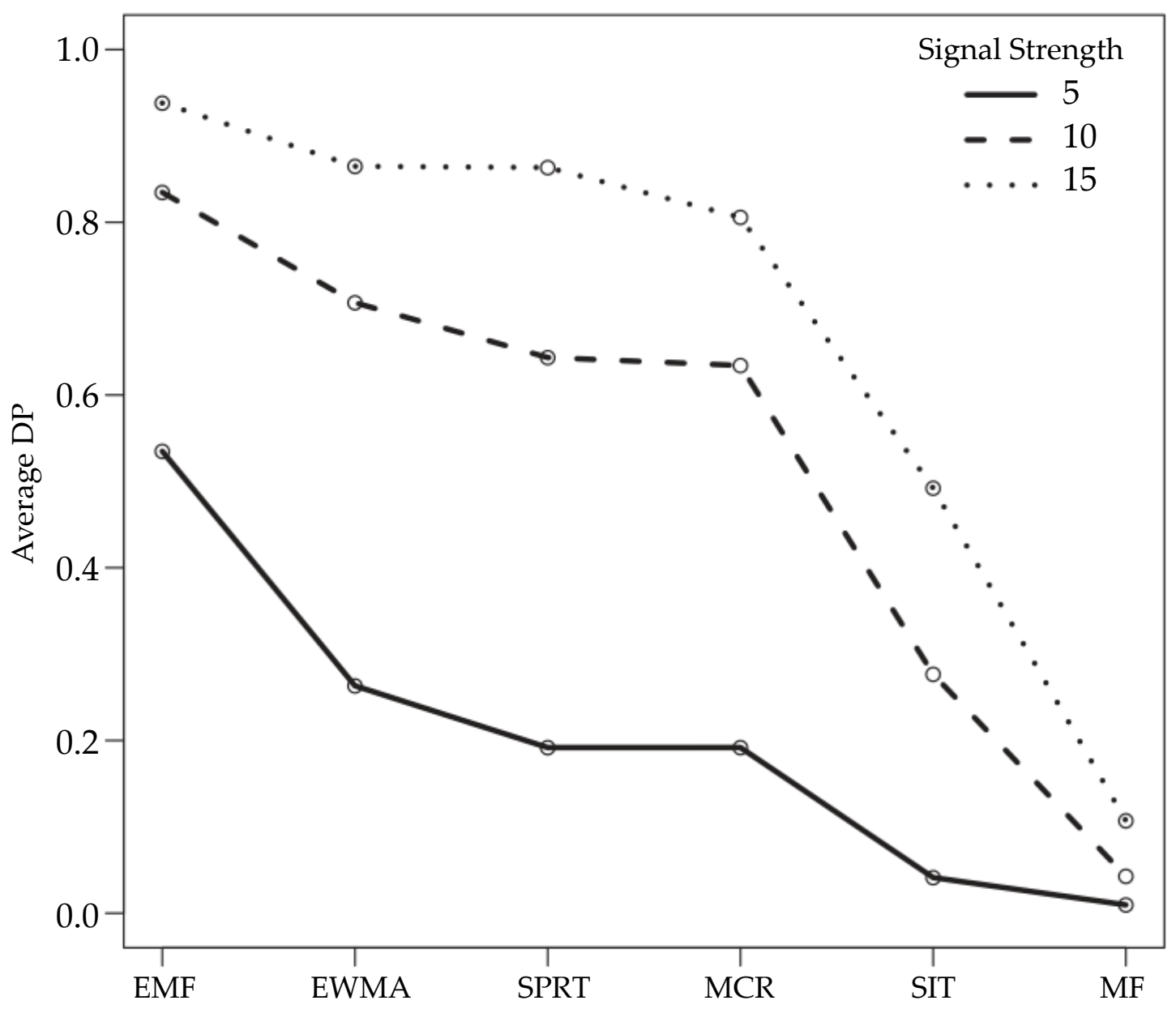

Figure 6. Comparison of the data processing methods estimated matched filtering (EMF), exponentially weighted moving average (EWMA), sequential probability ratio test (SPRT), maximum count rate (MCR), single-interval testing (SIT) and matched filter (MF) based on a Poisson model of neutron background and source counts, from [66]. Detection probabilities (DP or TPR) are averaged over twenty-four scenarios evaluated with the FPR set at 0.01 . The signal strength refers to the counts of the modeled sources as a multiple of the modeled background counts. Reprinted, with permission, from [66]. (C) 2013 American Statistical Association, reprinted by permission of Taylor \& Francis Ltd, on behalf of American Statistical Association.

\subsection{High Energy Resolution Scintillator Radiation Portal Monitors}

RPMs represent an optimal screening system for maintaining the high throughput requirement necessary at seaports, hence they are likely to remain a popular choice for non-intrusive shipping container inspection. Consequently, a challenge is to adapt the RPM system to possess the enhanced capabilities required of future screening systems without significantly increasing cost or impacting processing rates. The use of scintillating materials with higher energy resolution (as opposed to PVT) is one means that this might be achieved, although this requires a drop in the price of the necessary large, single crystal, inorganic scintillators, or the development of novel low-cost yet high energy resolution materials. Dual-mode scintillators may also comprise part of the solution by alleviating the additional costs of installing and operating separate neutron and $\gamma$ detection systems.

RPMs with spectroscopic capabilities have been demonstrated using $\mathrm{NaI}(\mathrm{Tl})$ and $\mathrm{LaBr}_{3}(\mathrm{Ce})$ scintillators (alongside separate neutron detectors) and are commercially available $[58,83]$, but their widespread implementation in the primary screening phase is prevented by their higher cost than PVT-based RPMs [13,26]. For these materials to be implemented in a single primary screening phase, developments in crystal growing techniques are required to greatly reduce costs. Alternatively, an increase in the security budget apportioned to installing more capable screening systems at seaports would enable 
the widespread implementation of commercially available spectroscopic RPMs that are currently beyond the financial viability of most ports. Other solutions may arise from advancements in the field of low-cost scintillator science, with new scintillators, ranging from nanomaterials to ceramics, constantly under development and existing materials such as Bi-loaded PVT already commercially available [41].

\section{Emerging Methods for Non-Portal-Based Shipping Container Screening \\ 5.1. Spatially Distributed Detector Networks}

Rather than utilising a few radiation detectors in small area, such is the case with an RPM measuring radiation in a small section of a traffic lane, an alternative approach is the adoption of a network of detectors spatially distributed across a larger area, with the use of their collective measurements to inspect objects passing through the area. The authors of [71] have demonstrated the benefits of such a detector network over the individual treatment of detector measurements using mathematical methods, simulation and experiment. For shipping container screening, there are various locations during the transportation of the container where a detector network could be employed for inspecting the radioactive contents of a container. One such place is in the same location where the current screening systems are situated (e.g., entrances, exits and other bottlenecks) giving a large sensitive region (IRSS C11 dataset uses detectors in an $\sim 40 \times 40 \mathrm{~m}$ array as shown in Figure $7 \mathrm{~b}$ [71]) as opposed to the smaller sensitive region between the RPM panels in the current system. Alternatively, a detector network could be built into a container ship, where measurement times on the order of weeks could be achieved during the voyage of the ship, facilitating accurate source characterisation and localisation. On a larger scale, a detector network could be spread across an entire seaport, although due to the size of seaports an unfeasible number of stationary detectors would likely be necessary to achieve the required sensitivity and, instead, a combination of stationary and mobile detectors could be used to continuously map the radiation environment of the port and identify illicit radioactive or nuclear material using advanced localisation and identification algorithms.

(a)

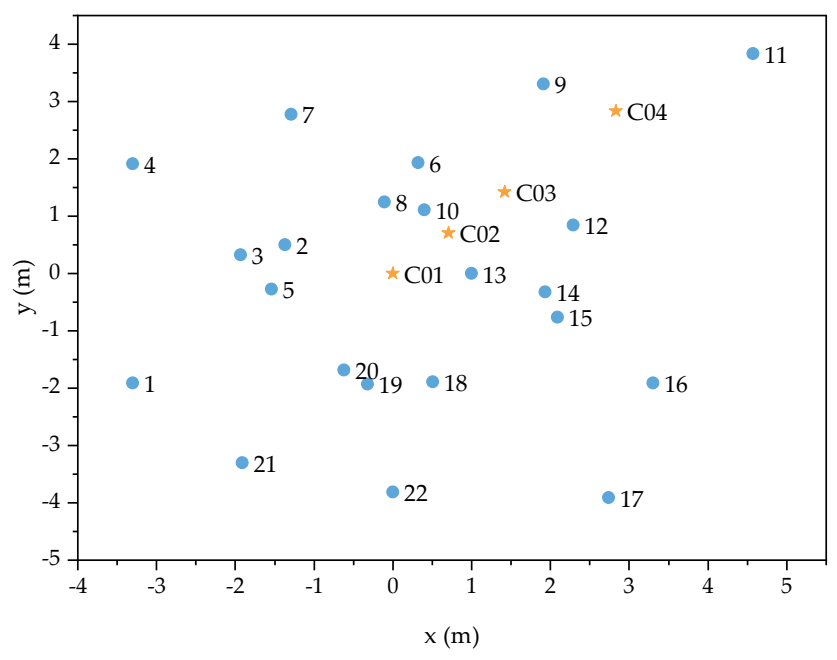

(b)

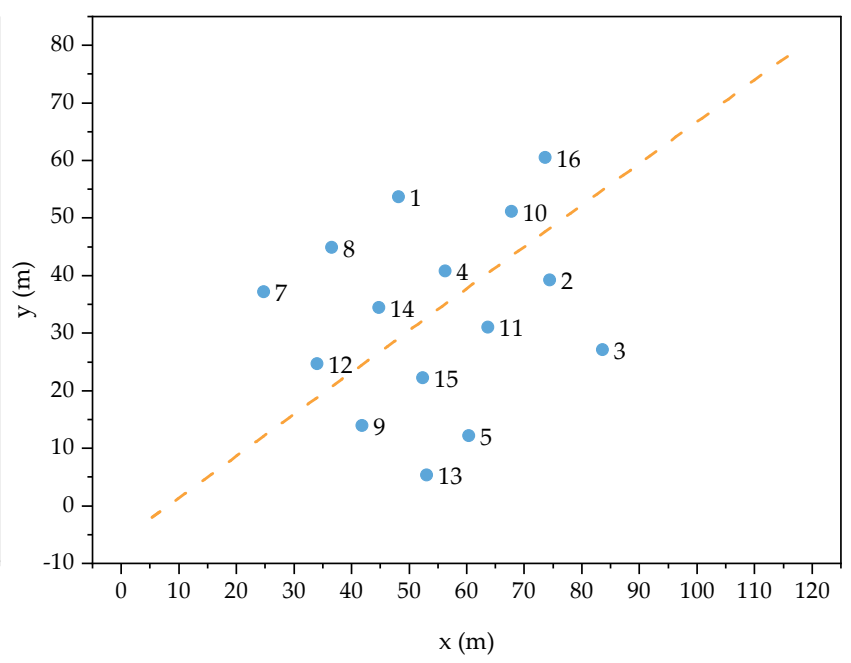

Figure 7. (a) Diagram illustrating the IRSS LSI_C indoor dataset. The blue circles indicate the locations of twenty-two $5.08 \times 5.08 \mathrm{~cm}$ cylindrical $\mathrm{NaI}(\mathrm{Tl})$ detectors, with orange markers showing the positions of a $281 \mathrm{kBq}{ }^{137} \mathrm{Cs}$ source in experiments LSI_C01, LSI_C02,LSI_C03 and LSI_C04 [71]. (b) Schematic of the IRSS C11 outdoor dataset experimental setup, with the blue circles indicating the locations of sixteen $5.08 \times 5.08 \mathrm{~cm}$ cylindrical $\mathrm{NaI}(\mathrm{Tl})$ detectors and the orange line defining the trajectory of a $6.5 \mathrm{MBq}{ }^{137} \mathrm{Cs}$ source [71].

Distributed detector networks also have applications outside of shipping container screening scenarios, as the systems are easily adaptable to pedestrian monitoring scenarios. Typically proposed detector networks use inorganic scintillators in each detector node, 
where small (low-geometric-efficiency) detectors are compensated for by the large sensitive region of the network, achieved through the dense spatial distribution of detectors over an area $[84,85]$. The use of smaller inorganic scintillator detectors introduces the additional benefits of allowing the systems to be deployed discretely and making them easily adaptable through the movement of the detectors. In addition, inorganic scintillators such as $\mathrm{NaI}(\mathrm{Tl})$ or $\mathrm{LaBr}_{3}(\mathrm{Ce})$ gives the detector network spectroscopic capability, enhancing its ability to differentiate between legitimate and nuisance events-generating alarms as necessary.

When processing the data measured by a network of detectors, a range of algorithms can be used to determine the presence of a source, with more sophisticated algorithms estimating the source location and activity. One such algorithm uses maximum likelihood estimation (MLE) to estimate the unknown parameters describing a source (e.g., position, activity) by modelling the expected counts at each detector from sources defined across the parameter space, and finding the source parameters for which the likelihood of the measured counts is the greatest [86].

Considering Poisson distributed counts defined by Equations (5) and (6), then ignoring attenuation for simplicity, the probability of measuring $x$ counts at a detector is given by;

$$
\mathbb{P}(x \mid \lambda)=\frac{\exp \left(-\left(\lambda_{b}+\lambda_{s}\right)\right)\left(\lambda_{b}+\lambda_{s}\right)^{x}}{x !}
$$

which allows the log likelihood function for $N$ detectors to be defined as

$$
\ell\left(\lambda_{0}, \mathbf{r}_{0}, \lambda_{b}\right)=\sum_{i=1}^{N}\left[x_{i} \log \left(\frac{\lambda_{0}}{d_{i}^{2}}+\lambda_{b}\right)-\left(\frac{\lambda_{0}}{d_{i}^{2}}+\lambda_{b}\right)-\log \left(x_{i} !\right)\right]
$$

where $\lambda_{0}$ and $\lambda_{b}$ are defined as in Equation (6), and $\mathbf{r}_{0}$ is the source position vector, such that $d_{i}=\left\|\mathbf{r}_{i}-\mathbf{r}_{0}\right\|$ where $\mathbf{r}_{i}$ is the position of the $i^{\prime}$ th detector $[67,86]$. The task then becomes finding the values of $\lambda_{0}, \mathbf{r}_{0}$ and $\lambda_{b}$ which maximise the log likelihood function, $\ell\left(\lambda_{0}, \mathbf{r}_{0}, \lambda_{b}\right)$.

Gradient algorithms are used by iteratively taking steps along the increasing gradient of $\ell$, however, this method may identify local maxima in $\ell$ as opposed to the desired global maximum [64]. To overcome this problem, a method proposed by [64] generates an initial estimate of the parameters in the neighbourhood of the global maximum after which iterative algorithms, such as a gradient algorithm, can be used to identify the global maximum.

Alternatively, a grid search method can be used which involves discretising the parameter space into grids and evaluating $\ell$ for each grid point, defined by $\left(\lambda_{0}, \mathbf{r}_{0}, \lambda_{b}\right)$; the values of $\ell$ can then be compared to find the global maximum $[67,87]$. As the grids become smaller (the parameter space is split into a finer mesh), the computational cost of this algorithm increases due to the increased number of calculations required, therefore, reducing the error associated with the source parameter estimates is increasingly processor intensive. A solution to this problem rationalises that for a given $\mathbf{r}_{0}$ the parameters $\lambda_{0}$ and $\lambda_{b}$ that maximise $\ell\left(\lambda_{0}, \lambda_{b}\right)$ can easily be computed [67]. This results in the maximum $\ell\left(\lambda_{0}, \lambda_{b}\right)$ being calculated for all possible $\mathbf{r}_{0}$, with the maximum corresponding to the most likely source location; the effect of this is to reduce the number of calculations required in the MLE algorithm, allowing the required localisation error to be achieved at less computational cost.

A maximum likelihood method has been used by [88] to process data from a network of seven $\mathrm{NaI}(\mathrm{Tl})$ detectors to estimate the location of a $189 \mathrm{kBq}{ }^{137} \mathrm{Cs}$ source in a $5 \times 5 \times 5 \mathrm{~m}$ parameter space [88]. In this method, the computational cost of this algorithm was reduced using a grid reduction method; in each iteration the grids were split into smaller grids (higher discretisation), but only the twenty grids with the highest likelihoods were used in the subsequent iteration, with the total number of calculations in each iteration resultantly remaining constant. As a measure of the likelihood, the intensity of a source at each grid point was calculated for each detector using the counts measured in each detector and the 
inverse square relationship, then, the standard deviation of the source intensity at each grid point is calculated, with a smaller standard deviation corresponding to a higher likelihood. This method was able to locate the source to within $0.6 \mathrm{~m}$ in under 3 mins of measurement, however, the authors predict that at $\sim 37 \mathrm{MBq}$ source activities the methodology could localise the source within only $1 \mathrm{~s}$.

Another algorithm for source detection and localisation uses triangulation, which relies on the assumption that;

$$
x_{i}(t)=\frac{\lambda_{0}}{d_{i}^{2}},
$$

where $x_{i}(t)$ is the number of counts in the $i^{\prime}$ th detector at time $t$, with $\lambda_{0}$ and $d_{i}$ as previously defined [89]. For a system of three detectors $(i \in\{1,2,3\})$ at known positions $\left(\mathbf{r}_{i}\right)$, the set of equations defined by Equation (12) is solved to estimate the source location, $\mathbf{r}_{0}$. When this method is applied, the location estimate assumes that the source lies within the convex hull of the detector arrangement and non-physical (imaginary roots) and misleading (multiple real roots) solutions must be rejected [89]. In the case where more than three detectors are used, Equation (12) can be solved for each subset of three detectors and the clustering of the source location estimates indicates the presence of a source.

One further algorithm, which is similar to triangulation, is a source-attractor radiation detection (SRD) method [89]. This approach uses a network of (n) detectors split into subsets of $k=3$ detectors. First, the geometric centroid of each sub-set is calculated yielding $\left(\begin{array}{l}n \\ k\end{array}\right)$ original centroid positions. Then, a shifted centroid is calculated for each sub-set by obtaining the point of intersection of the lines $\overline{\left(\mathbf{r}_{2}, d_{13}\right)}$ and $\overline{\left(\mathbf{r}_{3}, d_{12}\right)}$, where $d_{13}$ and $d_{12}$ are the count weighted centres of the lines between detectors 1, 2 and 1, 3, respectively-with $\mathbf{r}_{i}$ the position of the $i$ 'th detector. From this, a fixed radius enclosing circle is then defined at the location which maximises the number of shifted centroids within it. The radius of the enclosing circle is an important variable parameter in this method and for the IRSS C11 datasets radii between 1.0 and $1.5 \mathrm{~m}$ produced comparable performance across all runs [89]. By calculating the number of detector counts corresponding to the increased number of centroids (shifted minus original number) in the enclosing circle and comparing this to a threshold set using the desired TPR and FPR rates, the presence of a source is inferred.

Both triangulation radiation source detection (TriRSD) and SRD methods do not attempt to accurately localise sources, but rather infer the presence of a source from the clustering of source location estimates made by multiple subsets of detectors, thus their performances are assessed by detection TPR and FPR, rather than the localisation error. The TPR and FPR values for source detection achieved by TriRSD, SRD, MLE and a SPRT method were compared by [89], by implementing each algorithm on IRSS datasets LSI, C11 and B14, which together describe both moving and stationary ${ }^{137}$ Cs sources with differing configurations of detectors in indoor and outdoor environments-with the C11 and LSI_C setups shown in Figure 7. The first notable finding was the computation time of each algorithm, with SRD and SPRT making decisions in less than $10 \%$ of the measurement interval $(<0.1 \mathrm{~s})$, whereas decision times for the other methods varied from $0.34 \pm 0.05 \mathrm{~s}$ (TriRSD) to $0.83 \pm 0.09 \mathrm{~s}$ (MLE). This delay has important implications for real time detection that necessitates the algorithms derive decisions on whether to raise an alarm in less time than it takes to acquire a measurement.

The plot shown in Figure 8 illustrates the average detection (TPR) and false alarm (FPR) probabilities of the aforementioned methods averaged over all datasets. In addition to their faster computation, SRD and SPRT also posses the highest TPR values, 0.978 and 0.976 , respectively, although, SPRT is unfavourable due to its high false alarm rate (0.156) [89]. Clearly, SRD represents the best performing algorithm, whereas MLE, with the lowest TPR and the longest time to decision, performs the worst. However, MLE yields an estimate of the source location and its activity, representing a distinct advantage over the other algorithms that are only capable of making binary alarm decisions or in the case of TriSRD and SRD, localising sources that lie within the convex hull of three detectors. 


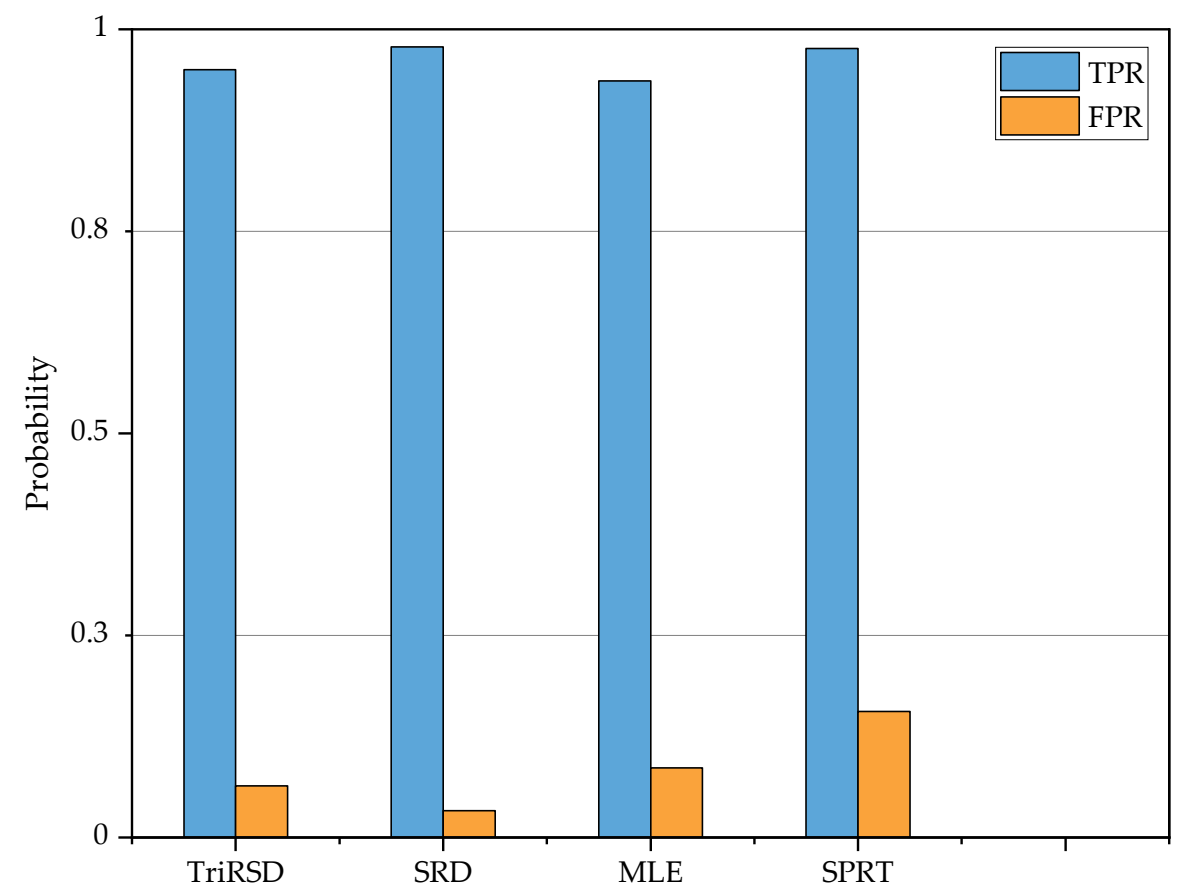

Figure 8. Average detection (TPR) and false alarm probabilities (FPR) for different algorithms applied to six IRSS datasets. Reprinted, with permission, from [89]. (C) 2021 IEEE.

A particle filter (PF) is a Bayesian method that uses Monte Carlo techniques and, like MLE, is also capable of producing source location and activity estimates [90,91]. In a PF, particles, each of which describes a possible source location and activity and a background count rate $\left(\lambda_{0}, \mathbf{r}_{0}, \lambda_{b}\right)$, are randomly sampled and assigned weightings. The weightings are calculated by taking measurements of the system and subsequently using them to estimate the likelihood of the particles. In each iteration of the PF, particles with weightings below a threshold are replaced by resampling and, consequently, the algorithm converges to identify the particles with the highest weightings. The resampling method effects the convergence properties of the algorithm, but in at least one case of radioactive source localisation the algorithm has been shown to converge using the simplest resampling method; drawing new particles from the original uniform distribution [92]. An example of a more elaborate resampling method, which may converge faster, resamples new particles from the distribution defined by the particles with weightings above the threshold.

Following the work by [92], the weight of a particle can be expressed as

$$
w_{k}^{i}=\sum_{j=1}^{d} \log \left(\frac{\lambda_{j}\left(p_{k}^{i}\right)^{x_{k}^{j}} \exp \left(-\lambda_{j}\left(p_{k}^{i}\right)\right)}{x_{k}^{j} !}\right)
$$

where $w_{k}^{i}$ is the weight of the $i^{\prime}$ th particle calculated from the $k^{\prime}$ th measurements, $\lambda_{j}\left(p_{k}^{i}\right)$ is the modelled response of the $j^{\prime}$ th detector from particle $p_{k}^{i}$ and $x_{k}^{j}$ is the $k^{\prime}$ th measurement at the $j$ 'th detector. Here, the Poisson assumption of counts (Equation (5)) and Equation (6) is used to calculate the modelled detector response, initially ignoring attenuation. Particles are then randomly sampled from the uniform space defined by $[\mathbb{X} \times \mathbb{Y} \times \mathbb{A}]$, where $[\mathbb{X} \times \mathbb{Y}]$ is the $2 \mathrm{D}$ cartesian coordinate space and $[\mathbb{A}]$ is the activity range to be searched. With subsequent measurements of the system, the algorithm is iterated and the weights in Equation (13) are recalculated; if a source is present the particles are expected to converge/cluster around the true source parameters, otherwise the particles stay dispersed [90]. The source parameters and their corresponding errors can be estimated from the location of the particle cluster and the degree of clustering, respectively. To decide whether this source constitutes a threat, the likelihood of the detector measurements given these source 
parameters can be calculated using Equation (11) and compared to threshold values that are set by the desired TPR and FPR. Particle filter methods have been demonstrated to work in scenarios where multiple radioactive sources are present or, importantly for implementation at a busy and high throughput seaport, scenarios with continuously moving radioactive sources $[90,93]$.

The authors of [92] applied a PF algorithm to the IRSS LSI_C stationary source datasets shown in Figure 7a, which was seen to converge if the source lies within the convex hull of the detector arrangement and the particles are sampled from a uniform distribution bounded in $[\mathbb{X} \times \mathbb{Y}]$ by this region [92]. For a $281 \mathrm{kBq}{ }^{137} \mathrm{Cs}$ source, this PF algorithm showed convergence to one or more clusters within the $120 \mathrm{~s}$ measurement period, and by simulation of additional data, it was shown that the source could be localised to within $1.5 \mathrm{~m}$ in under $5 \mathrm{~min}$ of measurement for all the IRSS LSI_C datasets [92].

In another study, the authors of [90] applied a PF to the measurements taken by detectors on the periphery of the IRSS C11 dataset, by excluding detectors $4,11,14$ and 15 in Figure $7 \mathrm{~b}$. They demonstrated that the PF was able to detect the moving $6.5 \mathrm{MBq}$ ${ }^{137} \mathrm{Cs}$ source while it transited the convex hull of the detector arrangement, as well as for several meters either side of it. Additionally, the PF was applied to individual or pairs of detector measurements to determine the systems capability when the detectors were not networked. Notably, it was demonstrated that even detectors located far from the source trajectory, which did not detect the source when their measurements were considered alone, contributed to the detection when the PF algorithm was used with the detector network [90].

For the detection of a radioactive source using a detector network, many of the aforementioned methods show significant promise for implementation to shipping container screening. The methods (TriRSD, SRD, MLE and SPRT) in Figure 8, for which the TPR and FPR values are averaged over multiple IRSS datasets, are capable of making binary alarm decisions based on measured data in under $1 \mathrm{~s}$, which is important for processing sequences of measurements as they are taken in real-time. For the IRSS C11 dataset, which comprises measurements of a source moving through a widespread array of detectors outdoors, which may be similar to the detector network scenario posed by a busy port, the TPR values of the methods TriRSD, SRD, MLE and SPRT are consistently below the averages shown in Table 4. In comparison, a PF acting on the same IRSS C11 dataset detected the presence of the source for the duration of of the time it was in the convex hull of the detector arrangement, indicating a better performance (in TPR) than the TriSRD, SRD, MLE and SPRT algorithms. However, there was no assessment of the FPR of this PF algorithm [90].

Table 4. Comparison of algorithms used to process measurements taken by a spatially distributed radiation detector network.

\begin{tabular}{llcccc}
\hline Method & Validation Dataset & TPR & FPR & Localisation & Refs. \\
\hline MLE & $\begin{array}{l}\text { 3 min of measurement of a } 189 \mathrm{kBq}^{137} \mathrm{Cs} \text { source } \\
\text { using 7 NaI(T1) detectors in a } 5 \times 5 \times 5 \text { m volume. }\end{array}$ & 1.0 & Unknown & Within 0.6 $\mathrm{m}$ & {$[88]$} \\
MLE & IRSS LSI, C11, B14. & 0.986 & 0.086 & - & {$[89]$} \\
\hline SPRT & IRSS LSI, C11, B14. & 0.976 & 0.156 & - & {$[89]$} \\
\hline TriRSD & IRSS LSI, C11, B14. & 0.950 & 0.064 & - & {$[89]$} \\
\hline SRD & IRSS LSI, C11, B14. & 0.978 & 0.033 & - & {$[89]$} \\
\hline PF & 5 min of IRSS LSI_C data. & 1.0 & Unknown & Within $1.5 \mathrm{~m}$ & {$[92]$} \\
PF & Periphery detectors in IRSS C11. & 1.0 & Unknown & - & {$[90]$} \\
\hline
\end{tabular}

At the cost of taking long sequences of measurements (on the order of minutes), MLE and PF algorithms both have the ability to localise weak ( $\sim 10 \mu \mathrm{Ci})$ stationary sources, to within $1.5 \mathrm{~m}$ for the source-detector configurations discussed in this review, as shown in Table 4 [88,92]. This is promising in scenarios where long measurement times on 
stationary containers are possible, such as would be the case with a detector network built into a container ship or a port-wide detector network. However, the cost of such a system associated with the large, dense array of detectors required is likely to prevent its implementation.

A more promising means of meeting the requirement of long measurement times on stationary shipping containers is to install detectors into port lifting equipment, such as the cranes used to unload container ships, where the detectors and container are in close proximity and stationary relative to one another for extended periods $(\sim 60 \mathrm{~s})$ during a lifting process [94]. Measurements taken in such a scenario with twenty or forty foot shipping containers, with dimensions $2.43 \times 2.59 \times 6.06 \mathrm{~m}$ and $2.43 \times 2.59 \times 12.19 \mathrm{~m}$ [95], respectively, would be on similar distance scales to the LSI_C dataset and the experimental work listed as item one in Table 4, where localisation within $1.5 \mathrm{~m}$ was achieved. Incorporating detectors into port lifting equipment therefore represents a scenario that would facilitate the use of MLE and PF algorithms to localise radioactive sources and introduces the additional possibility of screening discrete sections of a container by controlling the field of view of the detectors as they are lowered onto the top of a container. One further benefit of implementing detectors embedded in port lifting equipment is the reduced background count rate above water that results in an increased signal-to-noise ratio for measurements of a radioactive source taken over water when compared to measurements of the same source taken over the solid ground constituting the port [96]. Previous investigation into incorporating radiation detectors into port lifting equipment undertaken by [94] identified intrinsic limitations to such a system arising from the one-sided nature of the screening process, though it was recognised that performance may be improved through the use of advanced data processing algorithms. Further work is required to determine the change in background count rate above a waterborne container ship and whether the advantages from increased measurement time, minimal stand-off distance and increased signal-tonoise of such a detection system are sufficient to outweigh the disadvantages from taking measurements on only one side of a shipping container.

Due to their ability to localise sources, MLE and PF algorithms used with detector networks are likely form a part of the next generation of shipping container screening systems, however, further work is required to characterise the false alarm rates in such systems and minimise the localisation error. In order to constrain a source to a single shipping container, the localisation error is required to be significantly less than the shortest dimension of a shipping container, which is $2.43 \mathrm{~m}$ for standard twenty and forty foot containers. To improve the performance of detector networks at localising radioactive sources concealed within shipping containers, measurement times need to be maximised or higher-efficiency detector networks used, either by employing more detectors in the network or increasing the efficiency (e.g., size) of individual detectors.

The ANSI standards specify that a spectroscopic portal monitor should detect a $\sim 592 \mathrm{kBq}{ }^{137} \mathrm{C}$ s source moving through it, which is significantly below the activity used in the IRSS C11 measurements. While the detector networks used in this analysis are not portal monitors, one would expect them to meet similar performance benchmarks if this type of detector network were to replace existing inspection infrastructure [97]. Fortunately, the previously identified algorithms do not incorporate the spectroscopic capability of the $\mathrm{NaI}(\mathrm{Tl})$ detectors into the decision making process, which if used in parallel with these algorithms, would improve the performance (in TPR and FPR) of a detector networkbased screening system for detecting isotopes with characteristic photopeaks such as ${ }^{137} \mathrm{Cs}$. Additional work is hence required to determine the optimum performance capability of a detector network (such as that in IRSS C11), combining the count processing algorithms discussed in this section with algorithms that utilise the energy distribution measured by the detectors to inform alarm decisions. 


\subsection{Mobile Detector Networks}

In order to increase the sensitivity of a detector network distributed over a large area using a limited number of detectors, some (or all) of the detectors can be made mobile to form an adaptable network that can be cost-effectively implemented over a large search area such as a seaport [98]. Interest in this research area is demonstrated by the Detecting Radiological Threats in Urban Environments challenge set in 2019 by the US National Nuclear Security Administration (NNSA), with a prize pool of $\$ 100,000$ [99]. Using Monte Carlo methods, the NNSA simulated a single $5.08 \times 10.16 \times 40.64 \mathrm{~cm} \mathrm{NaI}(\mathrm{Tl})$ detector moving through model urban environments containing different sources such as HEU, WGPu, ${ }^{131} \mathrm{I},{ }^{60} \mathrm{Co}$ or ${ }^{99 \mathrm{~m}} \mathrm{Tc}$, asking research teams to design detection algorithms to identify the presence of sources, identify the isotopes and locate the point of closest approach of the detector $[99,100]$. A further demonstration of the interest in mobile detector networks is the USA Defence Advanced Research Projects Agency's (DARPA) SIGMA project in which over 1000 portable radiation detectors were used to map an urban area in Washington, D.C. $[101,102]$.

In a shipping container-filled seaport, detectors could be made mobile by attaching them to port vehicles, which are increasingly automated and have their movement patterns and accessible locations defined by the port design and operational procedures [103]. Much of the literature around mobile detector networks is based on urban environments where the detector movement is restricted to roads [67,92,104]; this might impose similar constraints as to those on detectors mounted on port vehicles moving around shipping container stacks, which are typically arranged in a grid formation, as shown in Figure 9, not dissimilar to roads in an urban environment. To control detector motion, commonly proposed mobile detector networks use either randomly moving detectors or base the detector motion in subsequent time intervals on the detector measurements made in the previous time interval to direct the detector towards regions of higher radiation $[67,105]$. At a seaport, the predetermined detector path along the route of the task performed by a port vehicle may restrict the sensitivity of a mobile detector network; whether these detector paths provide the required coverage for the network to be effective is likely to be port specific and requires further investigation, though on first inspection the problem seems to be analogous to radiation detectors traversing roads in an urban environment.

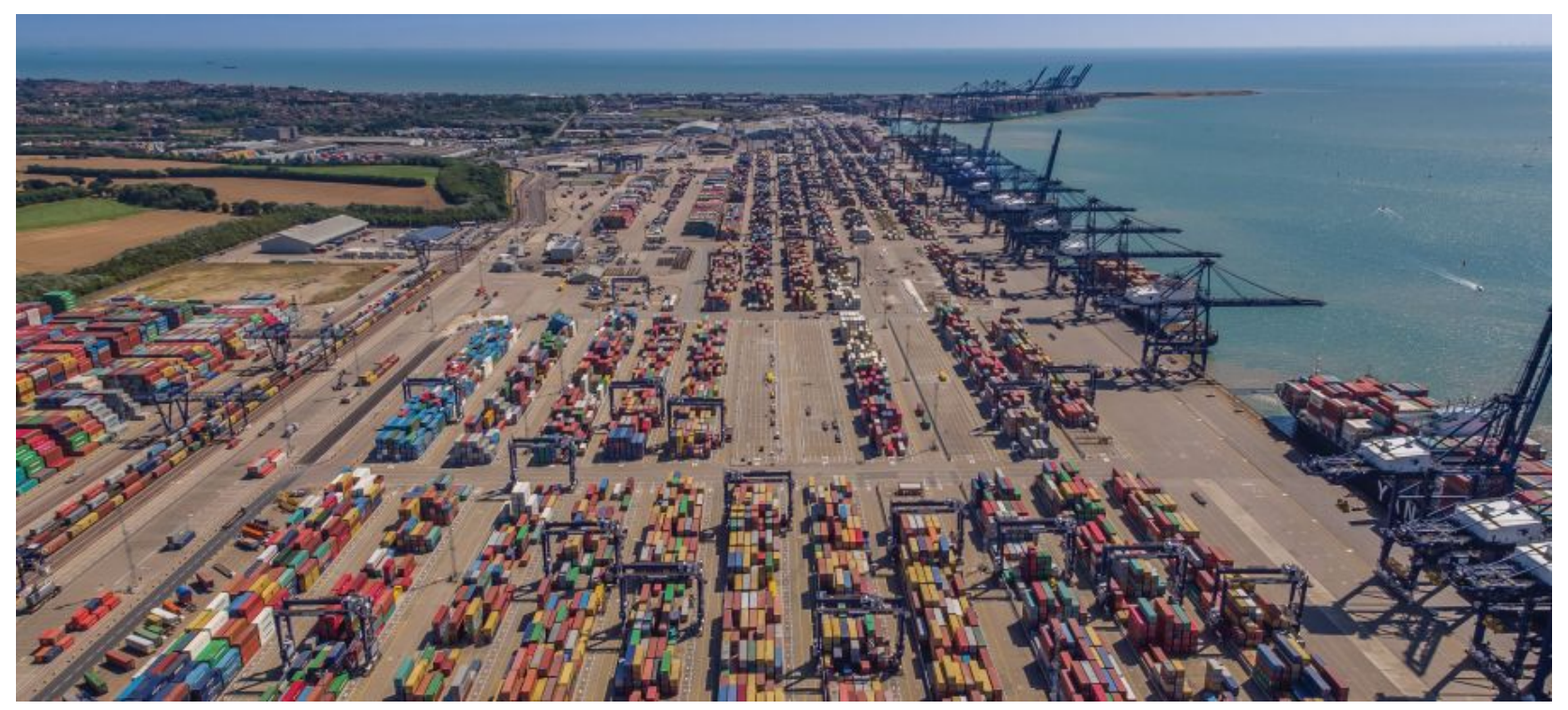

Figure 9. Grid layout of container stacks at Felixstowe Port, UK. Photograph reprinted, with permission, courtesy of the Port of Felixstowe. (C) 2021 Port of Felixstowe.

The authors of [67] used a maximum likelihood method to detect and estimate the locations of four $\sim 44 \mathrm{MBq}{ }^{54} \mathrm{Mn}$ sources in a simulated urban environment compris- 
ing thirty-six $(100 \times 80 \mathrm{~m})$ blocks/buildings separated by $10 \mathrm{~m}$ wide roads. With fifty $(13.2 \times 8.0 \times 2.35 \mathrm{~mm}) \mathrm{CsI}(\mathrm{Tl})$ detectors randomly moving along the roads, a grid search method was able to localise the sources to within $2 \mathrm{~m}$ in $\sim 200 \mathrm{~s}$. This method was also applied to experimental background measurements taken in a $450 \times 350 \mathrm{~m}$ urban area with modelled ${ }^{137} \mathrm{Cs}$ source profiles injected into the data at different distances from the background detector measurement path. The results of this study are shown in Figure 10. For localising sources within the width of a shipping container $(\sim 2.43 \mathrm{~m})$, only large (74 MBq) sources could be identified at $10 \mathrm{~m}$ distances from the background measurement positions, although for $5 \mathrm{~m}$ distances, sources as low as $18.5 \mathrm{MBq}$ could be localised to within $2.5 \mathrm{~m}$ [67]. For both the modelled data and the background measurements with injected source profiles, the large search areas involved in this study suggest this method may be transferable to a port scenario as well as the urban environment; although the performance of this system for detecting weaker sources requires further investigation and refinement.

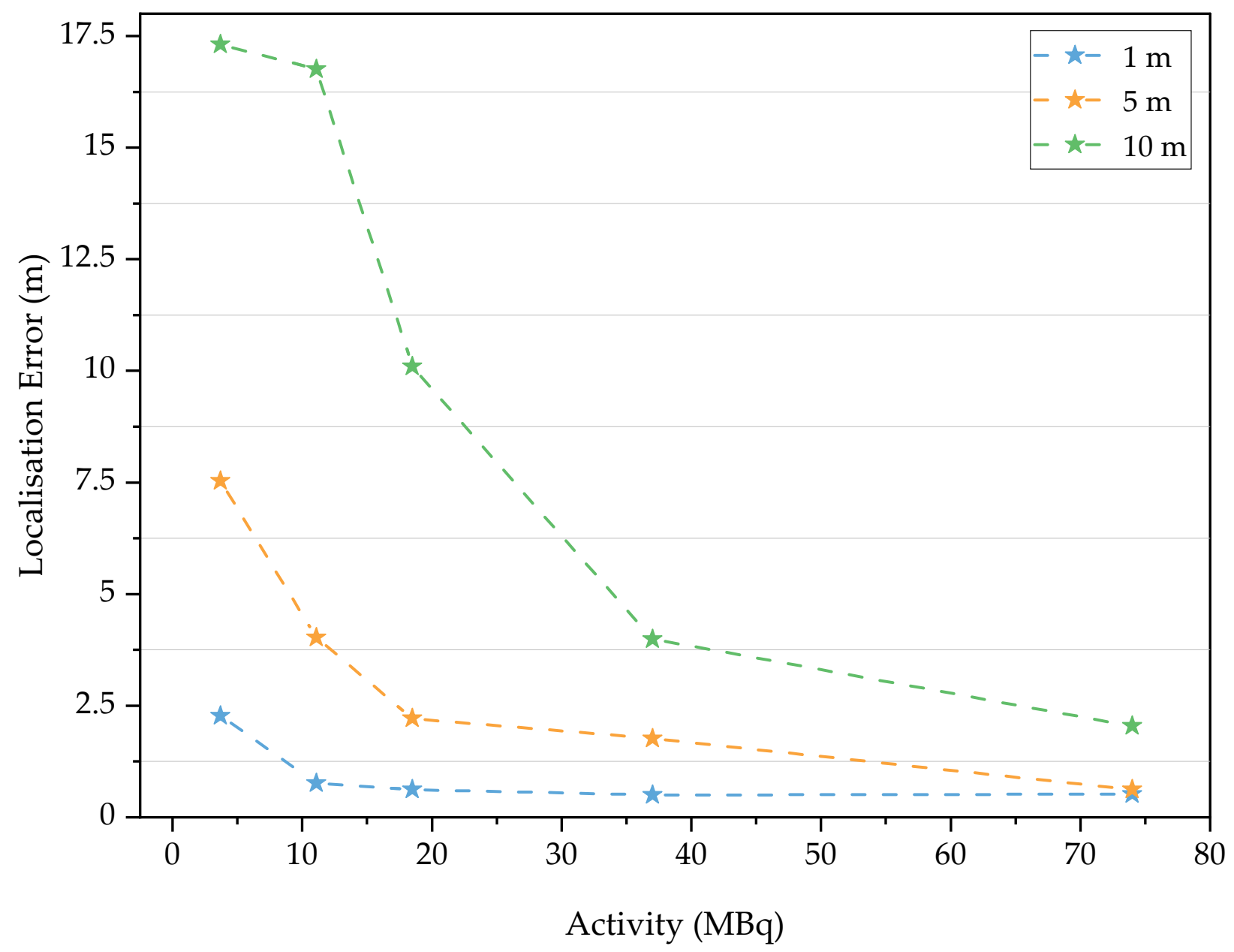

Figure 10. Source localisation errors for various activity source profiles injected into background measurements taken in a $450 \times 350 \mathrm{~m}$ urban area. Injected source profiles correspond to a ${ }^{137} \mathrm{Cs}$ source at distances of 1,5 or $10 \mathrm{~m}$ from the background measurement positions. Results from [67].

PF algorithms were also used to process data from simulated mobile detector networks distributed across urban environments [92,93]. The authors of [92] tested one method on a mathematical model of a $322 \mathrm{MBq}$ source in two different locations within a 2-D representation of a $250 \times 180 \mathrm{~m}$ urban environment, wherein different blocks (representing buildings) in the 2-D map were assigned optical thicknesses to simulate the associated $\gamma$ attenuation [92]. At each step, detectors progressed towards the mean position of the 
non-resampled particles without leaving the boundaries of the mobile area (roads). Using this method, with 100 measurement steps, the particles were shown to cluster around the source location, with the resultant localisation error on the order of metres [92].

In another deployable PF method investigated by [93], one or more detectors made searches around an area, choosing in which direction to move and for how long to acquire measurements at the next location based on the optimum estimated information gain predicted from prior measurements. Using Monte Carlo simulations, this method was able to identify multiple sources with one or more moving detectors and demonstrated the superior performance of an information driven search over both random and uniform methods in key performance benchmarks, such as search time and localisation error. Based on experimental measurements of two $(\sim 37 \mathrm{MBq}){ }^{137} \mathrm{Cs}$ sources in a large outdoor area $\left(\sim 10,000 \mathrm{~m}^{2}\right)$, this algorithm was shown to localise both sources to within $3.13 \mathrm{~m}$ in an average time of $93.4 \mathrm{~s}$ using a single mobile Geiger-Müller detector, ignoring detector movement time [93]. The resultant implication for seaport shipping container screening applications is that for detectors in a network moving on paths defined by port operations, the optimum performance is unlikely to be achieved owing to the inability of such a system to investigate high radiation fields away from the predefined detector paths. From a more positive standpoint, this result suggests that the performance of such a mobile detector network could be significantly enhanced through the introduction of one or more detectors that employ an adaptable information-driven approach to radiation mapping.

Other algorithms used alongside mobile detectors to detect illicit radioactive sources are discussed by $[98,104]$. In the former, the search area was split into grids and a graph theory method was used to localise $\gamma$-emitting sources. For mobile networks, the MLE, PF and graph-theory methods discussed yield source estimate locations which, as formerly alluded to, are a key property for future screening systems and can contribute to intelligent detector movement that greatly improves algorithm performance [93]. The drawback of source localisation methods, however, is the computational power required for performing these algorithms. To produce a real-time radiation map of a seaport, the detector network based screening system would rely on a powerful central node to receive data, perform calculations and temporally and spatially align the results from individual detectors [106]. On the other hand, the implementation of such a mobile detector network at a seaport may be facilitated by the fact that a port represents a well-defined search area and detectors could be mounted on automated port vehicles which already have their locations tracked in real time as part of port operational procedures [103].

\subsection{Intelligent Data Fusion Methods}

Fusion of passive radiation measurements of a shipping container with other relevant data, such as a description of a containers contents or the location of a shipping container, can lead to improved screening systems by aiding source localisation and activity estimates, which ultimately serve to increase TPR and decrease FPR values. By characterising the contents of a shipping container, either through the use of manifest information (an inventory of the goods being transported in the container) or X-ray radiography, the attenuation term in Equation (6) can be more appropriately accounted for [68,107]. Improving the accuracy of the attenuation term in Equation (6) represents a means of enhancing the capability of SPRT, MLE and PF algorithms that require the calculation of detector responses to different radiation sources, which otherwise would rely on assumptions on the degree of attenuation owing to the variable nature of cargo contents.

A method that combines $\mathrm{NaI}(\mathrm{Tl}) \mathrm{RPM}$ data with X-ray radiography and manifest information to characterise the activity of NORM cargo was proposed by [107]. Firstly, a sequence of RPM measurements were used with background suppression and detector field of view models to calculate the spatial distribution of the activity (counts) in the cargo for specific energy regions of interest (ROIs). These energy ROIs were selected based on manifest information and for NORM cargo were chosen to be centred around $1460 \mathrm{keV}\left({ }^{40} \mathrm{~K}\right), 1765 \mathrm{keV}\left({ }^{214} \mathrm{Bi}\right.$ in the ${ }^{238} \mathrm{U}$ decay chain) and $2614 \mathrm{keV}\left({ }^{212} \mathrm{Bi}\right.$ in the ${ }^{232} \mathrm{Th}$ 
decay chain) $[27,107]$. Subsequently, by using radiography to estimate the density and volume fraction of cargo in the container, the activity of the isotopes in the cargo could be estimated by solving Equation (6) for $\lambda_{0}$ in each energy ROI, thus characterising the NORM cargo. This method serves to aid the accurate identification of NORMs, reducing nuisance alarms while also contributing to knowledge of NORM cargo characteristics for use in simulations [107].

The authors of [68] proposed another method to identify and localise sources concealed in shipping containers using X-ray radiography to derive a 3-D model of the container material characteristics by back-projecting individual pixels from a radiographic image of the container to generate voxels. The isotopic composition of the voxels were taken to be a weighted average of the materials (e.g., container walls, container floor, cargo) and the density of each voxel was calculated from the grey scale value of the pixel in the radiograph. Then, using an MLE grid search method, the source location and activity were estimated taking into account the attenuation from the voxels. Using this method, an estimation of the source localisation error was made to circa $40 \mathrm{~cm}$ (for a $370 \mathrm{kBq}{ }^{133} \mathrm{Ba}$ source), although the source activity estimates were more sensitive to estimated material density, assumed composition and the radiative scattering model used. The limiting factor for the computation time of this method was the calculation of the source-detector responses that are used to calculate the likelihoods in the MLE grid search. Their approach calculated the field of expected responses for each detector such that the radiation transport only had to be calculated once for each container. For 124 effective plastic scintillator detectors this calculation took $\sim 15$ mins on a 312 node cluster, which therefore rules out this method for real-time or primary screening. However, significant reductions in computation time were achieved by ignoring scattering in the calculations, at the cost of reducing the accuracy of the source activity estimates [68].

While the benefits of using X-ray radiography to characterise the contents of a shipping container are clear, it is unlikely to form a viable component of future systems for shipping containers screening to identify the existence of concealed radioactive or nuclear material. Firstly, it would necessitate an additional inspection system to generate the radiographic images, increasing the operational and financial cost of the system. Secondly, the benefits of accurately accounting for attenuation by describing the cargo composition and geometry introduce more complexity to the calculations and thus increase the computational cost of the method, which limits the ability of the method to yield alerting decisions in real-time without the use of high performance computers.

An alternative method relies on data fusion to correlate the motion of a shipping container, measured using vision sensors or position tracking, with changes in radiation measurements $[108,109]$. Fortunately, as shipping containers each posses unique identification numbers, branding and markings, as well as being stacked and transported around the port site in a methodical and coordinated way, the position of a shipping container at any point in time should be attainable from the port systems that order the movement and arrangement of containers in a port [103]. This method, therefore, is promising for implementation in a seaport environment with a spatially distributed stationary or mobile detector network, as the shipping container motion data used to correlate with radiation measurements is already being recorded.

One method, by [108], performed mathematical models of vehicles taking discrete steps along one of eight traffic lanes to determine the feasibility of a motion correlation method in an international border monitoring scenario. They proposed tracking vehicle locations with either video surveillance or mandatory radio-frequency identification (RFID) devices in each vehicle and use the vehicle location data to correlate the number of times radiation detector measurements change with the movement of the vehicles in steps along the traffic lane. To identify the vehicle most likely to contain a radioactive source, the vehicle that corresponded to changes in radiation measurements most frequently was taken, or an inverse square law relationship was fitted to the graph of radiation measurements against vehicle position for each vehicle, with the vehicle corresponding to the best fit most likely 
to contain the radioactive source. The authors demonstrated that both the methods for identifying the vehicle containing the source converged to the correct vehicle, under the condition that the source strength was over 250 times greater than background (to ensure that there is a detectable change in detector counts when the vehicles step between the two furthest measurement positions) [108]. In reality, this is not a practicable condition and this detection system may not be suitable for detecting low-activity or shielded sources. However, the stepping motion of vehicles is analogous to shipping containers being shifted in stacks and so a variant of this method could merit further investigation for application at a seaport with a network of radiation detectors.

\section{Conclusions}

For the non-intrusive screening of shipping containers for illicit radioactive or nuclear materials at a seaport, plastic scintillator-based RPMs are widely deployed to make binary decisions on whether a container requires a secondary, more thorough, inspection. Since a shipping container can be screened in transit on the back of a flatbed truck using PVT RPMs, the primary screening process has a high throughput and at the cost of low energy resolution, achieves reasonable sensitivity through the high geometric efficiency associated with the large sizes of PVT panels available. Despite the low energy resolution of PVT plastic scintillators, false-positive alarm events can be minimised in PVT RPMs by implementing data processing algorithms, such as EW, which provide a level of discrimination between legitimate threats and nuisance radiation sources by considering the shapes of the spectra expected from different types of materials, such as NORMs and SNMs. Ultimately, though, the primary screening capability of a PVT RPM is fundamentally limited by the poor energy resolution of the PVT volumetric detectors, which inhibit its ability to properly characterise the source composition, activity and location. Consequently, a slow secondary screening phase is required to validate alarms triggered in the primary phase and characterise and locate illicit sources that may be present, costing operational resources and causing delays.

Resulting from the very real threat of nuclear terrorism and the global increase in nuclear materials available as the world adopts a greater nuclear "backbone" through such low carbon energy sources, a more robust shipping container screening system is required. The next generation of shipping container screening systems should be capable of performing a thorough inspection on every container in a single screening phase with a high throughput, such that false alarms become non-existent and all significant concealed sources are identifiable with high probability.

One of the key aspects of a screening system is the radiation detector material that it utilises, dictating the sensitivity of the system to different energies and types of radiation, the number and arrangement of detectors required and the compatibility with different data processing algorithms. Currently, the choice for a detector system can be crudely simplified to high-geometric-efficiency, low-cost and low energy resolution plastic scintillators or low-geometric-efficiency, high-cost and high energy resolution inorganic scintillators. The reason for the widespread implementation of the former results from the higher sensitivity that can be achieved at a lower comparable cost, therefore, the ongoing development of new, economically advantageous scintillating materials or inorganic crystal growing techniques has the potential to revolutionise shipping container screening systems $[13,26]$.

Networks of high-resolution inorganic scintillator radiation detectors, either mobile or stationary, represent a possible means of implementing a single-phase shipping container screening system with source characterisation and localisation capability. In this review, multiple studies of such detector networks, designed for use in urban and border monitoring scenarios, which are capable of localising radioactive sources have been identified. To apply detector networks to a seaport, detectors could be mounted on port vehicles that move around stationary container stacks and continuously map the radiation environment of the port. Alternatively (or additionally), detectors could be attached to port infrastructure to form a widespread stationary detector network, although this would have to be restricted to a small section of a port that acts as a bottleneck, or perhaps a 
container ship itself, for the number of detectors required to be feasibly low. Another potential implementation of inorganic scintillator detector networks is in shipping container transport infrastructure, such as lifting equipment or automated flatbed vehicles, which would enable the individual inspection of containers as they are inherently positioned at bottleneck locations as each shipping container must be loaded and unloaded from a container ship and transported around the port. All these implementations of detector networks merit further research to assess their feasibility and compatibility with day-to-day seaport procedures, as they must not inhibit normal seaport operation and will require data fusion with the operational database of a seaport to identify specific shipping containers and account for their movement in day-to-day port operation.

Whatever the choice of radiation detector material and configuration, a data processing algorithm is crucial to extract the maximum amount of information from the potentially limited detector measurements in order to determine if there exists concealed radioactive or nuclear materials in the cargo and, where possible, perform characterisation. For simply detecting the presence of sources, the use of the most complex algorithms such as MLE and $\mathrm{PF}$ is unnecessary as similar performance is achievable with simple algorithms, such as the counts above threshold criteria with improvements based on the energy spectrum measured by a detector. However, for the next generation of detection systems to posses source tracking or source position and activity estimation capabilities, more complex algorithms such as MLE and PF are necessary. These advanced algorithms are suited to screening systems where the shipping container remains in the sensitive region of the detectors for extended time periods (on the order of minutes for the methods compared in this review), such that sufficient measurements can be taken for the algorithms to suitably converge to an estimate of a well-defined source. To achieve the required measurement times for every shipping container passing through a port using current RPM-based systems would cause huge delays, and hence the benefit of using spatially distributed detector networks that take measurements on shipping containers as they are processed through the port is evident.

From this review, areas of further research key to the development of the next generation of shipping container screening systems for characterising and localising illicit radioactive or nuclear material can be identified. By experimental measurement or simulation the feasibility of implementing inorganic scintillator detectors, such as in mobile networks or lifting equipment, in a seaport environment should be further investigated. While research into such inorganic scintillator-based detection systems has been conducted in the past $[71,94,96]$ and despite the fact that they are commercially available [110], RPMs remain the predominant form of radiation detection infrastructure in place at seaports. However, through the use of existing and emerging advanced data processing algorithms and by exploiting the properties of novel inorganic scintillator materials, there is potential for such mobile detector networks or detectors embedded into lifting equipment to become feasible alternatives to RPMs. Future investigations should, therefore, aim to establish limits on the sensitivity of these systems using algorithms such as MLE, PF or other advanced algorithms not discussed in this work.

Ultimately, combining this information with the isotopic characterisation that is possible as a result of using high energy resolution detectors, the performance of these systems in key benchmarks such as FPR, TPR, localisation error and decision time should be identified and compared to ascertain an optimum detector configuration and algorithm combination. An analysis of the transshipment of containers from ship to road, or vice versa, may also identify new potential implementations of detection systems with properties, such as exposure time, which ideally suit different detector types and additionally allow for higher-resolution measurements.

Author Contributions: Investigation, original draft preparation, review and editing, E.L.C.; review and editing, P.G.M. Both authors have read and agreed to the published version of the manuscript.

Funding: This research received no external funding. 


\section{Data Availability Statement: Not applicable.}

Acknowledgments: The authors wish to thank the support provided by the Nuclear Security Network (NuSec) for EC's PhD, with the funding from AWE additionally acknowledged.

Conflicts of Interest: The authors declare no conflict of interest, financial or otherwise.

\section{References}

1. Downes, R.J. Alarming Cargo: Regulation and Control at the UK Border. Terror. Political Violence 2019, 33, 549-571. [CrossRef]

2. Ferguson, C.D.; Potter, W.C.; Spector, L.S.; Sands, A.; Potter, W.C. The Four Faces of Nuclear Terrorism; Florence: Taylor \& Francis Group: Oxfordshire, UK, 2005

3. International Atomic Energy Agency. Combating Illicit Trafficking in Nuclear and other Radioactive Material; IAEA Nuclear Security Series No. 6; IAEA: Vienna, Austria, 2007.

4. Janssens-Maenhout, G.; De Roo, F.; Janssens, W. Contributing to shipping container security: Can passive sensors bring a solution? J. Environ. Radioact. 2010, 101, 95-105. [CrossRef]

5. Hamrashdi, H.A.; Monk, S.D.; Cheneler, D. Passive gamma-ray and neutron imaging systems for national security and nuclear non-proliferation in controlled and uncontrolled detection areas: Review of past and current status. Sensors 2019, $19,2638$. [CrossRef]

6. Glasstone, S.; Dolan, P.J.; United States Department of Defense. The Effects of Nuclear Weapons, 3rd ed.; Dolan, P.J., Glasstone, S., Eds.; U.S. Dept. of Defense: Washington, DC, USA, 1977; 653p.

7. Ferguson, C.D. Reducing the threat of RDDs. IAEA Bull. 2003, 45, 12-15.

8. Rosoff, H.; Von Winterfeldt, D. A risk and economic analysis of dirty bomb attacks on the ports of Los Angeles and Long Beach. Risk Anal. 2007, 27, 533-546. [CrossRef]

9. International Atomic Energy Agency. IAEA Incident and Trafficking Database (ITDB); IAEA: Vienna, Austria, 2020.

10. HM Government Department for Business, Energy and Industrial Strategy. Powering Our Net Zero Future; The Energy White Paper; The Stationery Office: London, UK, December 2020.

11. HM Government Department for Transport. UK Port Freight Statistics: 2019; Technical Report; Government of the United Kingdom: London, UK, 2020.

12. HM Government Home Office CONTEST: The United Kingdom's Strategy for Countering Terrorism; Technical Report; Government of the United Kingdom: London, UK, 2018.

13. Klann, R.T.; Shergur, J.; Mattesich, G. Current State of Commercial Radiation Detection Equipment for Homeland Security Applications. Nucl. Technol. 2009, 168, 79-88. [CrossRef]

14. Burr, T.; Gattiker, J.R.; Myers, K.; Tompkins, G. Alarm criteria in radiation portal monitoring. Appl. Radiat. Isot. 2007, 65, 569-580. [CrossRef] [PubMed]

15. Ely, J.; Kouzes, R.; Schweppe, J.; Siciliano, E.; Strachan, D.; Weier, D. The use of energy windowing to discriminate SNM from NORM in radiation portal monitors. Nucl. Instrum. Methods Phys. Res. Sect. A Accel. Spectrometers Detect. Assoc. Equip. 2006, 560, 373-387. [CrossRef]

16. Geelhood, B.D.; Ely, J.H.; Hansen, R.R.; Kouzes, R.T.; Schweppe, J.E.; Warner, R.A. Overview of portal monitoring at border crossings. In Proceedings of the 2003 IEEE Nuclear Science Symposium. Conference Record, Portland, OR, USA, 19-25 October 2008; pp. 513-517. [CrossRef]

17. Robinson, S.M.; Kaye, W.R.; Schweppe, J.E.; Siciliano, E.R. Optimal background attenuation for fielded radiation detection systems. IEEE Nucl. Sci. Symp. Conf. Rec. 2007, 54, 1277-1284. [CrossRef]

18. Keller, P.E.; Kouzes, R.T. Influence of extraterrestrial radiation on radiation portal monitors. IEEE Trans. Nucl. Sci. 2009, 56, 1575-1583. [CrossRef]

19. Kouzes, R.T.; Siciliano, E.R. The response of radiation portal monitors to medical radionuclides at border crossings. Radiat. Meas. 2006, 41, 499-512. [CrossRef]

20. Bendahan, J. Review of active interrogation techniques. Nucl. Instrum. Methods Phys. Res. Sect. A Accel. Spectrometers Detect. Assoc. Equip. 2020, 954, 161120. [CrossRef]

21. Armitage, J.; Bryman, D.; Cousins, T.; Gallant, G.; Jason, A.; Jonkmans, G.; Noël, S.; Oakham, G.; Stocki, T.J.; Waller, D.; et al. Cosmic Ray Inspection and Passive Tomography for SNM Detection. AIP Conf. Proc. 2009, 1194, 24-35 doi:10.1063/1.3275662. [CrossRef]

22. Thomay, C.; Velthuis, J.J.; Baesso, P.; Cussans, D.; Steer, C.; Burns, J.; Quillin, S.; Stapleton, M. A Novel Markov Random Field-Based Clustering Algorithm to Detect High-Z Objects With Cosmic Rays. IEEE Trans. Nucl. Sci. 2015, 62, 1837-1848. [CrossRef]

23. Liew, S.V. Can active proton interrogation find shielded nuclear threats at human-safe radiation levels? Nucl. Instrum. Methods Phys. Res. Sect. A Accel. Spectrometers Detect. Assoc. Equip. 2017, 855, 47-54. [CrossRef]

24. Knoll, G.F. Radiation Detection and Measurement, 4th ed.; John Wiley \& Sons, Inc.: Hoboken, NJ, USA, 2010.

25. Berger, M.J.; Hubbell, J.H.; Seltzer, S.M.; Chang, J.; Coursey, J.S.; Sukumar, R.; Zucker, D.S.; Olsen, K. XCOM: Photon Cross Sections Database. National Institute of Standards and Technology, Gaithersburg, MD. Available online: https://www.physics. nist.gov/PhysRefData/Xcom/html/xcom1.html (accessed on 22 March 2021). 
26. Milbrath, B.D.; Peurrung, A.J.; Bliss, M.; Weber, W.J. Radiation detector materials: An overview. J. Mater. Res. 2008, 23, 2561-2581. [CrossRef]

27. Gilmore, G.R. Practical Gamma-Ray Spectrometry, 2nd ed.; John Wiley \& Sons, Ltd.: Chichester, UK, 2008; Volume 52, p. 379. [CrossRef]

28. Lilley, J. Nuclear Physics: Principles and Applications; Wiley: Chichester, UK, 2001.

29. Ely, J.H.; Siciliano, E.R.; Kouzes, R.T. Comparison of $\mathrm{NaI}(\mathrm{Tl})$ scintillators and high purity germanium for vehicle portal monitor applications. In Proceedings of the IEEE Symposium Conference Record Nuclear Science, Rome, Italy, 16-22 October 2004; pp. 1584-1587. [CrossRef]

30. Eljen. GENERAL PURPOSE EJ-200, EJ-204, EJ-208, EJ-212. Available online: https://eljentechnology.com/products/plasticscintillators / ej-200-ej-204-ej-208-ej-212 (accessed on 25 January 2021).

31. Eljen Technology. Neutron/Gamma PSD EJ-301, EJ-309. Available online: https://eljentechnology.com/products/liquidscintillators / ej-301-ej-309 (accessed on 7 July 2021).

32. Advatech UK. NaI(Tl)—Sodium Iodide (Tl). Available online: https://www.advatech-uk.co.uk/nai_tl.html (accessed on 21 January 2021).

33. Saint-Gobain. Scintillation Crystal Materials. Available online: https://www.crystals.saint-gobain.com/products/crystalscintillation-materials (accessed on 25 January 2021).

34. Advatech UK. CsI(Tl)—Cesium Iodide (Thallium). Available online: https://www.advatech-uk.co.uk/csi_tl.html (accessed on 17 February 2021).

35. Advatech UK. LaBr3(Ce)—Lanthanum Bromide (Ce) Scintillator Crystal. Available online: https://advatech-uk.co.uk/labr3_ce. html (accessed on 25 January 2021).

36. Advatech UK. LYSO(Ce)_Lutetium Yttrium Orthosilicate (Ce). Available online: https://advatech-uk.co.uk/lyso_ce.html (accessed on 25 January 2021).

37. Pepin, C.M.; Berard, P.; Perrot, A.; Pepin, C.; Houde, D.; Lecomte, R.; Melcher, C.L.; Dautet, H. Properties of LYSO and recent LSO scintillators for phoswich PET detectors. IEEE Trans. Nucl. Sci. 2004, 51, 789-795. [CrossRef]

38. Advatech UK. CLYC(Ce)—Cesium Lithium Yttrium Chloride (Ce). Available online: https://advatech-uk.co.uk/clyc_ce.html (accessed on 25 January 2021).

39. Woolf, R.S.; Wulf, E.A.; Phlips, B.F.; Chowdhury, P.; Jackson, E.G. Identification of internal radioactive contaminants in elpasolites (CLYC, CLLB, CLLBC) and other inorganic scintillators. Nucl. Instrum. Methods Phys. Res. Sect. A Accel. Spectrometers Detect. Assoc. Equip. 2020, 954, 161228. [CrossRef]

40. Cherepy, N.J.; Martinez, H.P.; Beck, P.R.; Sanner, R.D.; Hok, S.; Drury, O.B.; Swanberg, E.L.; Payne, S.A.; O'Neal, S.P.; Hurlbut, C.R. Bismuth-loaded plastic scintillator portal monitors. In Proceedings of the SPIE 10762, Hard X-ray, Gamma-Ray, and Neutron Detector Physics XX, 107620B, San Diego, CA, USA, 13 September 2018; doi:10.1117/12.2320884. [CrossRef]

41. Dujardin, C.; Auffray, E.; Bourret-Courchesne, E.; Dorenbos, P.; Lecoq, P.; Nikl, M.; Vasil’Ev, A.N.; Yoshikawa, A.; Zhu, R.Y. Needs, trends, and advances in inorganic scintillators. IEEE Trans. Nucl. Sci. 2018, 65, 1977-1997. [CrossRef]

42. Pawełczak, I.A.; Glenn, A.M.; Martinez, H.P.; Carman, M.L.; Zaitseva, N.P.; Payne, S.A. Boron-loaded plastic scintillator with neutron- $\gamma$ pulse shape discrimination capability. Nucl. Instrum. Methods Phys. Res. Sect. A Accel. Spectrometers Detect. Assoc. Equip. 2014, 751, 62-69. [CrossRef]

43. Shirwadkar, U.; van Loef, E.; Markosyan, G.; Tower, J.; Spens, M.; Ji, C.; Pandian, L.S.; Gueorguiev, A.; Glodo, J.; Shah, K.; et al. Low-cost, multi-mode detector solutions. Nucl. Instrum. Methods Phys. Res. Sect. A Accel. Spectrometers Detect. Assoc. Equip. 2020, 954, 161289. [CrossRef]

44. Glodo, J.; Wang, Y.; Shawgo, R.; Brecher, C.; Hawrami, R.H.; Tower, J.; Shah, K.S. New Developments in Scintillators for Security Applications. Phys. Procedia 2017, 90, 285-290. [CrossRef]

45. D'Olympia, N.; Chowdhury, P.; Jackson, E.G.; Lister, C.J. Fast neutron response of 6Li-depleted CLYC detectors up to $20 \mathrm{MeV}$. Nucl. Instrum. Methods Phys. Res. Sect. A Accel. Spectrometers Detect. Assoc. Equip. 2014, 763, 433-441. [CrossRef]

46. Chadwick, M.B.; Obložinský, P.; Herman, M.; Greene, N.M.; McKnight, R.D.; Smith, D.L.; Young, P.G.; MacFarlane, R.E.; Hale, G.M.; Frankle, S.C.; et al. ENDF/B-VII.0: Next Generation Evaluated Nuclear Data Library for Nuclear Science and Technology. Nucl. Data Sheets 2006, 107, 2931-3060. [CrossRef]

47. Nie, Q.; Zhu, J.; Ma, H.; Zeng, Z.; Cheng, J.; Li, J.; Zhang, H. Neutron/Gamma-ray Pulse Shape Discrimination with a CLYC Scintillator. In Proceedings of the 2019 IEEE Nuclear Science Symposium and Medical Imaging Conference, NSS/MIC 2019, Manchester, UK, 26 October-2 November 2019; doi:10.1109/NSS/MIC42101.2019.9059774. [CrossRef]

48. Lubsandorzhiev, B.K. On the history of photomultiplier tube invention. Nucl. Instrum. Methods Phys. Res. Sect. A Accel. Spectrometers Detect. Assoc. Equip. 2006, 567, 236-238. [CrossRef]

49. Photomultiplier Tubes and Assemblies for Scinitllation Counting \& High Energy Physics. Available online: https://www. hamamatsu.com/eu/en/support/resources/index.html (accessed on 17 February 2021).

50. Donati, S.; Tambosso, T. Single-Photon Detectors: From Traditional PMT to Solid-State SPAD-Based Technology. IEEE J. Sel. Top. Quantum Electron. 2014, 20, 204-211. [CrossRef]

51. Marano, D.; Belluso, M.; Bonanno, G.; Billotta, S.; Grillo, A.; Garozzo, S.; Romeo, G.; Catalano, O.; Rosa, G.; Sottile, G.; et al. Silicon Photomultipliers Electrical Model Extensive Analytical Analysis. Nucl. Sci. IEEE Trans. 2014, 61, 23-34. [CrossRef] 
52. Buzhan, P.; Dolgoshein, B.; Filatov, L.; Ilyin, A.; Kantzerov, V.; Kaplin, V.; Karakash, A.; Kayumov, F.; Klemin, S.; Popova, E.; et al. Silicon photomultiplier and its possible applications. Nucl. Instrum. Methods Phys. Res. Sect. A Accel. Spectrometers Detect. Assoc. Equip. 2003, 504, 48-52. [CrossRef]

53. Kim, M.; Kang, H.; Kim, H.; Kim, W.; Park, H.; Kim, S. A Study of CsI(Tl) Scintillator with Optimized Conditions of Large Area Avalanche Photodiode. J. Nucl. Sci. Technol. 2008, 45, 586-589. [CrossRef]

54. Ikagawa, T.; Kataoka, J.; Yatsu, Y.; Saito, T.; Kuramoto, Y.; Kawai, N.; Kokubun, M.; Kamae, T.; Ishikawa, Y.; Kawabata, N. Study of large area Hamamatsu avalanche photodiode in a $\gamma$-ray scintillation detector. Nucl. Instrum. Methods Phys. Res. Sect. A Accel. Spectrometers Detect. Assoc. Equip. 2005, 538, 640-650. [CrossRef]

55. Hamamatsu Photonics, K.K. Hamamatsu Products. Available online: https://www.hamamatsu.com/eu/en/product/index.html (accessed on 22 January 2021).

56. Fawcett, T. An introduction to ROC analysis. Pattern Recognit. Lett. 2006, 27, 861-874. [CrossRef]

57. Live Chart of Nuclides. Available online: https://www-nds.iaea.org/relnsd/vcharthtml/VChartHTML.html (accessed on 27 January 2021).

58. Kouzes, R.T.; Ely, J.H.; Milbrath, B.D.; Schweppe, J.E.; Siciliano, E.R.; Stromswold, D.C. Spectroscopic and non-spectroscopic radiation portal applications to border security. In Proceedings of the IEEE Nuclear Science Symposium Conference Record, Fajardo, PR, USA, 23-29 October 2005; Volume 1, pp. 321-325. [CrossRef]

59. Southern Scientific Ltd. Guardian Radiation Portal Monitor Datasheet. Available online: https://www.southernscientific.co.uk/ products-by-application / security-cbrne-defence / radiation / portal-monitors / vehicle-portal-radiation-monitors? source=1550 \{\#\}downloads (accessed on 22 March 2021).

60. Stromswold, D.C.; Darkoch, J.W.; Ely, J.H.; Hansen, R.R.; Kouzes, R.T.; Milbrath, B.D.; Runkle, R.C.; Sliger, W.A.; Smart, J.E.; Stephens, D.L.; et al. Field tests of a NaI(Tl)-based vehicle portal monitor at border crossings. In Proceedings of the IEEE Symposium Conference Record Nuclear Science 2004, Rome, Italy, 16-22 October 2004; Volume 1, pp. 196-200. [CrossRef]

61. Armenteros, J.C.; Suárez, M.J.; Pujol, L. Dynamic numerical recipes to improve detection schemes for the interdiction of radioactive shipment. J. Radioanal. Nucl. Chem. 2018, 318, 865-875. [CrossRef]

62. Lo Presti, C.A.; Weier, D.R.; Kouzes, R.T.; Schweppe, J.E. Baseline suppression of vehicle portal monitor gamma count profiles: A characterization study. Nucl. Instrum. Methods Phys. Res. Sect. A Accel. Spectrometers Detect. Assoc. Equip. 2006, 562, $281-297$. [CrossRef]

63. Coulon, R.; Dumazert, J. Shadow-Shielding Compensation for Moving Sources Detection. IEEE Trans. Nucl. Sci. 2017, 64, 1641-1646. [CrossRef]

64. Bai, E.; Yosief, K.; Dasgupta, S.; Mudumbai, R. The maximum likelihood estimate for radiation source localization: Initializing an iterative search. In Proceedings of the 53rd IEEE Conference on Decision and Control, Los Angeles, CA, USA, 15-17 December 2014; pp. 277-282. [CrossRef]

65. Jarman, K.D.; Smith, L.E.; Carlson, D.K. Sequential probability ratio test for long-term radiation monitoring. IEEE Trans. Nucl. Sci. 2004, 51, 1662-1666. [CrossRef]

66. Burr, T.; Hamada, M.S. Moving neutron source detection in radiation portal monitoring. Technometrics 2013, 55, 296-308. [CrossRef]

67. Zhao, J.; Sullivan, C.J. Detection and parameter estimation of radioactive sources with mobile sensor networks. Radiat. Phys. Chem. 2019, 155, 265-270. [CrossRef]

68. Miller, E.A.; White, T.A.; Jarman, K.D.; Kouzes, R.T.; Kulisek, J.A.; Robinson, S.M.; Wittman, R.A. Combining radiography and passive measurements for radiological threat localization in Cargo. IEEE Trans. Nucl. Sci. 2015, 62, 2234-2244. [CrossRef]

69. Cooke, C.; Velthuis, J.J.; Beck, L.; Martin, P. A Novel Approach to Determining the Distance from a Source with a Single Measurement and no Prior Knowledge of Source Activity. In Proceedings of the 2019 IEEE Nuclear Science Symposium and Medical Imaging Conference, NSS/MIC 2019, Manchester, UK, 26 October-2 November 2019. [CrossRef]

70. Panaino, C.M.V.; Mackay, R.I.; Sotiropoulos, M.; Kirkby, K.J.; Taylor, M.J. Full 3D position reconstruction of a radioactive source based on a novel hyperbolic geometrical algorithm. Comput. Phys. Commun. 2020, 252, 107131. [CrossRef] [PubMed]

71. Rao, N.S.; Sen, S.; Berry, M.L.; Wu, Q.; Grieme, M.; Brooks, R.R.; Cordone, G. Datasets for radiation network algorithm development and testing. In Proceedings of the 2016 IEEE Nuclear Science Symposium, Strasbourg, France, 29 October-6 November 2016

72. Hevener, R.; Yim, M.S.; Baird, K. Investigation of energy windowing algorithms for effective cargo screening with radiation portal monitors. Radiat. Meas. 2013, 58, 113-120. [CrossRef]

73. Grisa, T.; Sas, D. On the ratio distribution of energy windowing algorithms for radiation portal monitors. Appl. Radiat. Isot. 2018, 132, 195-199. [CrossRef]

74. Paff, M.G.; Ruch, M.L.; Poitrasson-Riviere, A.; Sagadevan, A.; Clarke, S.D.; Pozzi, S. Organic liquid scintillation detectors for on-the-fly neutron/gamma alarming and radionuclide identification in a pedestrian radiation portal monitor. Nucl. Instrum. Methods Phys. Res. Sect. A Accel. Spectrometers Detect. Assoc. Equip. 2015, 789, 16-27. [CrossRef]

75. Paff, M.G.; Di Fulvio, A.; Clarke, S.D.; Pozzi, S.A. Radionuclide identification algorithm for organic scintillator-based radiation portal monitor. Nucl. Instrum. Methods Phys. Res. Sect. A Accel. Spectrometers Detect. Assoc. Equip. 2017, 849, 41-48. [CrossRef]

76. Lee, H.C.; Shin, W.G.; Park, H.J.; Yoo, D.H.; Choi, C.I.; Park, C.S.; Kim, H.S.; Min, C.H. Validation of energy-weighted algorithm for radiation portal monitor using plastic scintillator. Appl. Radiat. Isot. 2016, 107, 160-164. [CrossRef] [PubMed] 
77. Lee, H.C.; Choi, H.J.; Koo, B.T.; Choi, C.I.; Park, C.S.; Kim, H.S.; Min, C.H. Dynamic radionuclide identification using energy weighted algorithm with commercialized radiation portal monitor based on plastic scintillators. Radiat. Phys. Chem. 2020, 170, 108645. [CrossRef]

78. Coop, K.L. Monte carlo simulation of the sequential probability ratio test for radiation monitoring. IEEE Trans. Nucl. Sci. 1985, 32, 934-938. [CrossRef]

79. Fehlau, P.E. Comparing A Recursive Digital Filter with the Moving-Average and Sequential Probability-Ratio Detection Methods for SNM Portal Monitors. IEEE Trans. Nucl. Sci. 1993, 40, 143-146. [CrossRef]

80. Runkle, R.C.; Mercier, T.M.; Anderson, K.K.; Carlson, D.K. Point source detection and characterization for vehicle radiation portal monitors. IEEE Trans. Nucl. Sci. 2005, 52, 3020-3025. [CrossRef]

81. Ghelman, M.; Berdichevsky, V.; Vax, E.; Osovizky, A.; Marcus, E.; Ginzburg, D.; Gonen, E.; Mazor, T.; Cohen, Y. RPMs performances enhancement - Using matched filter algorithm and GLRT to estimate source activity and location. In Proceedings of the 2013 IEEE Nuclear Science Symposium and Medical Imaging Conference (2013 NSS/MIC), Seoul, Korea, 27 October2 November 2013; pp. 1-4. [CrossRef]

82. Lucas, J.M.; Saccucci, M.S. Exponentially Weighted Moving Average Control Schemes: Properties and Enhancements. Technometrics 1990, 32, 1-12. [CrossRef]

83. Yaar, I.; Peysakhov, I. A multiple-detector Radioactive Material Detection Spectroscopic (RMDS) portal. Nucl. Instrum. Methods Phys. Res. Sect. A Accel. Spectrometers Detect. Assoc. Equip. 2013, 712, 62-74. [CrossRef]

84. Rao, N.S.V.; Ma, C.Y.T.; Yau, D.K.Y. On performance of individual, collective and network detection of propagative sources. In Proceedings of the 2012 15th International Conference on Information Fusion, Singapore, 9-12 July 2012; pp. $247-254$.

85. Brennan, S.M.; Mielke, A.M.; Torney, D.C.; Maccabe, A.B. Radiation detection with distributed sensor networks. Computer 2004, 37, 57-59. [CrossRef]

86. Wan, H.; Zhang, T.; Zhu, Y. Detection and localization of hidden radioactive sources with spatial statistical method. Ann. Oper. Res. 2012, 192, 87-104. [CrossRef]

87. Cheng, Y.; Singh, T. Source term estimation using convex optimization. In Proceedings of the 2008 11th International Conference on Information Fusion, Cologne, Germany, 30 June-3 July 2008; pp. 1-8. [CrossRef]

88. Sharma, M.K.; Alajo, A.B.; Lee, H.K. Three-dimensional localization of low activity gamma-ray sources in real-time scenarios. Nucl. Instrum. Methods Phys. Res. Sect. A Accel. Spectrometers Detect. Assoc. Equip. 2016, 813, 132-138. [CrossRef]

89. Wu, C.Q.; Berry, M.L.; Grieme, K.M.; Sen, S.; Rao, N.S.; Brooks, R.R.; Cordone, G. Network Detection of Radiation Sources Using Localization-based Approaches. IEEE Trans. Ind. Inform. 2019, 15, 2308-2320. [CrossRef]

90. Rao, N.S.V.; Sen, S.; Prins, N.J.; Cooper, D.A.; Ledoux, R.J.; Costales, J.B.; Kamieniecki, K.; Korbly, S.E.; Thompson, J.K.; Batcheler, J.; et al. Network algorithms for detection of radiation sources. Nucl. Instrum. Methods Phys. Res. Sect. A Accel. Spectrometers Detect. Assoc. Equip. 2015, 784, 326-331. [CrossRef]

91. Ristic, B.; Arulampalam, S.; Gordon, N. Beyond the Kalman Filter: Particle Filters for Tracking Applications; Artech House: Boston, MA, USA, 2004

92. Cook, J.; Smith, R.C.; Ramirez, C.; Rao, N.S.V. Particle Filtering Convergence Results for Radiation Source Detection. arXiv 2020, arXiv:2004.08953.

93. Ristic, B.; Morelande, M.; Gunatilaka, A. Information driven search for point sources of gamma radiation. Signal Process. 2010, 90, 1225-1239. [CrossRef]

94. Ely, J.H.; Ashbaker, E.D.; Batdorf, M.T.; Baciak, J.E.; Hensley, W.K.; Jarman, K.D.; Robinson, S.M.; Sandness, G.A.; Schweppe, J.E. Spreader-Bar Radiation Detection System Enhancements: A Modeling and Simulation Study; Technical Report; Pacific Northwest National Lab. (PNNL): Richland, WA, USA, 2012.

95. Cleveland Containers. 20ft Shipping Containers. Available online: https://www.clevelandcontainers.co.uk/containers/20ftcontainers (accessed on 29 July 2021).

96. Grypp, M.D.; Marianno, C.M.; Poston, J.W.; Hearn, G.C. Design of a spreader bar crane-mounted gamma-ray radiation detection system. Nucl. Instrum. Methods Phys. Res. Sect. A Accel. Spectrometers Detect. Assoc. Equip. 2014, 743, 1-4. [CrossRef]

97. American National Standard for Performance Criteria for Spectroscopy-Based Portal Monitors Used in Homeland Security. Available online: https:/ / ieeexplore.ieee.org/document/7394937 (accessed on 29 July 2021).

98. Chandy, K.; Bunn, J.; Liu, A. Models and Algorithms for Radiation Detection. Modeling and Simulation Workshop for Homeland Security, 2010; pp. 1-6. Available online: https:// citeseerx.ist.psu.edu/viewdoc/download?doi=10.1.1.190.7783\&rep=rep1 \&type $=$ pdf (accessed on 29 July 2021)

99. Detecting Radiological Threats in Urban Areas. Available online: https:/ / www.topcoder.com/challenges/d616d145-d7e7-4d629323-9c558b216b40 (accessed on 14 December 2020).

100. Porter, M.D.; Akakpo, A. Detecting, identifying, and localizing radiological material in urban environments using scan statistics. In Proceedings of the 2019 IEEE International Symposium on Technologies for Homeland Security (HST), Woburn, MA, USA, 5-6 November 2019; pp. 1-6. [CrossRef]

101. Defense Advanced Research Projects Agency. Advanced Radioactive Threat Detection System Completes First Large-Scale Citywide Test. Available online: https:/ / www.darpa.mil/news-events/2016-10-11 (accessed on 10 May 2021).

102. Defense Advanced Research Projects Agency. SIGMA. Available online: https://www.darpa.mil/program/sigma (accessed on 10 May 2021). 
103. Vis, I.F.A.; De Koster, R. Transshipment of containers at a container terminal: An overview. Eur. J. Oper. Res. 2003, 147, 1-16. [CrossRef]

104. Hochbaum, D.S.; Fishbain, B. Nuclear threat detection with mobile distributed sensor networks. Ann. Oper. Res. 2011, 187, 45-63. [CrossRef]

105. Nakamura, Y.; Ito, M.; Sezaki, K. Planning Placement of Distributed Sensor Nodes to Achieve Efficient Measurement BT—Distributed, Ambient and Pervasive Interactions: Understanding Humans; Springer International Publishing: Cham, Switzerland, 2018; pp. 103-113.

106. Sullivan, C.J. Radioactive source localization in urban environments with sensor networks and the Internet of Things. In Proceedings of the 2016 IEEE International Conference on Multisensor Fusion and Integration for Intelligent Systems (MFI), Baden-Baden, Germany, 3-6 December 2016; pp. 384-388. [CrossRef]

107. Jarman, K.D.; Scherrer, C.; Smith, L.E.; Chilton, L.K.; Anderson, K.K.; Ressler, J.J.; Trease, L.L. Indirect estimation of radioactivity in containerized cargo. Radiat. Meas. 2011, 46, 10-20. [CrossRef]

108. Nicol, D.; Tsang, R.; Ammerlahn, H.; Johnson, M. Detection of Nuclear Material at Border Crossings using Motion Correlation. In Proceedings of the 2006 Winter Simulation Conference, Monterey, CA, USA, 3-6 December 2006; pp. 536-544. [CrossRef]

109. Stadnikia, K.; Martin, A.; Henderson, K.; Koppal, S.; Enqvist, A. Data-Fusion for a Vision-Aided Radiological Detection System: Sensor dependence and Source Tracking. EPJ Web Conf. 2018, 170, 6. [CrossRef]

110. Veritrainer Asset Holding. Veritrainer. Available online: http://veritainer.com/index.html (accessed on 13 May 2021). 